\title{
1 Knocking out AtCALS7 disturbs photoassimilate distribution and 2 weakens defence against phytoplasma infection.
}

3

4

Chiara Bernardini ${ }^{1}$, Amit Levy ${ }^{2}$, Sara Buoso ${ }^{1}$, Alberto Loschi ${ }^{1}$, Simonetta Santi ${ }^{1}$, Marta Martini ${ }^{1}$, Federico Bosetto ${ }^{1}$, Stacy Welker ${ }^{2}$, Joon Hyuk Suh ${ }^{3}$, Yu Wang ${ }^{3}$, Christopher Vincent ${ }^{4}$, Myrtho O'Pierre ${ }^{4}$, Aart J. E. van $\mathrm{Bel}^{5}$, Rita Musetti ${ }^{1 *}$

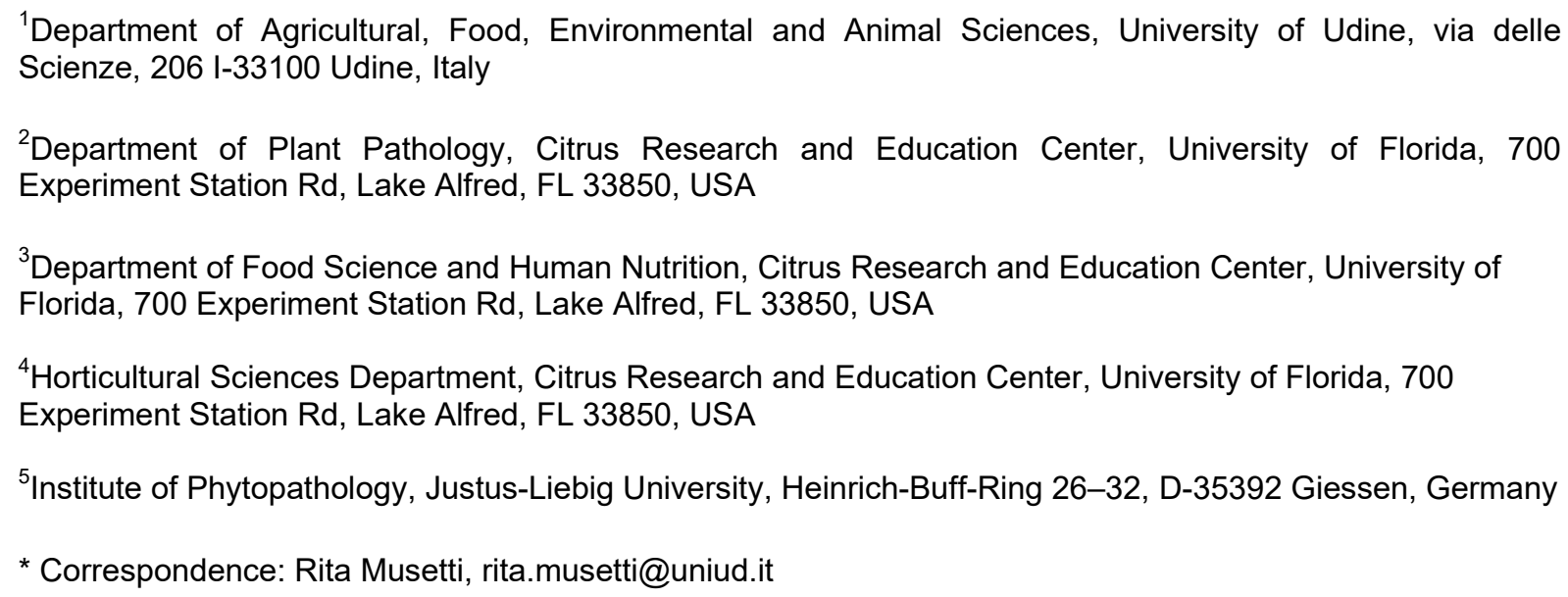

Key words: Arabidopsis, callose, defense, phloem, phytoplasma, sugar metabolism, transport

One sentence summary: Knocking out AtCALS7 disturbs photoassimilate distribution between axial and terminal sinks and weakens the defence against phytoplasma infection.

Running title: Arabidopsis cals7ko line and phytoplasma infection

\section{Author contribution}

CB and RM conceived the project. CB grew the plants, reared the insects and prepared inoculum sources with the help of $A L$ and FB. CB, SB and MM performed the molecular analyses. AmL conceived sugar quantification and the flow speed experiment with the support of CV. CB and SW performed confocal observations. CB, JHS and YW performed the sugar quantification assays. CB with the supervision of MOP performed the flow speed experiment. CB and RM and AJEvB wrote the manuscript with the valuable support of SS. All the authors provided critical suggestions for the realization of the manuscript. 


\section{ABSTRACT}

Callose accumulation around sieve pores, under control of Callose synthase 7 (AtCALS7), has been interpreted as a mechanical response to limit pathogen spread in phytoplasma-infected plants. AtCALS7 is also involved in sieve-pore development and, hence, in mass-flow regulation, carbohydrate metabolism and distribution, and plant growth. Multiple roles of AtCALS7 during phytoplasma infection were investigated in healthy and phytoplasma-infected [Chrysanthemum Yellows (CY)-phytoplasma] wild-type and Atcals7ko Arabidopsis lines. In keeping with an increased phytoplasma titre in Atcals7ko plants, floral stalk height of infected wild-type and mutant plants was reduced by, respectively, 88 and $100 \%$ in comparison to their healthy controls, suggesting a higher investment of host resources in phytoplasma growth in the absence of AtCALS7. The apparently increased susceptibility of mutants was investigated by microscopic, metabolic and molecular analyses. Infection influenced the sieve-pore functionality in wild-type plants, which hardly affected plant growth, and plasmodesmata in the cortex, a phenomenon less prominent in mutants. Infection also increased the level of some sugars (glucose, sucrose, myoinositol), but to the highest extent in mutants. Finally, infection induced a similar upregulation of gene expression of enzymes involved in sucrose cleavage (AtSUS5, AtSUS6) in mutants and wild-type plants and an upregulation of carbohydrate transmembrane transporters (AtSWEET11, AtSTP13, AtSUC3) in mutants only. A more effective plasmodesmal closure seemingly suppressed spread of phytoplasma effectors, which rendered wild-type plants less susceptible to infection, because gene expression of enzymes channeling carbohydrates towards phytoplasmas is less promoted.

\section{Introduction}

Phytoplasmas are wall-less, pleomorphic plant pathogens belonging to the class Mollicutes. They are confined to the sieve elements (SEs) of host plants (van Bel and Musetti, 2019) or to the body of phloem-feeding insect vectors (Alma et al., 2019). Phytoplasmas cause serious yield losses and affect the quality of crops of economic interest (Albertazzi et al., 2009). Profound alterations in transcriptome and proteome (Albertazzi et al., 2009; Cao et al., 2017; 2019), and in the phytohormone balance of 
infected plants (Dermastia, 2019; Bernardini et al., 2020) are reflected by a vast range of symptoms, such as witches'-brooms, leaf chlorosis, virescence, phyllody, and floral abortion (Ermacora and Osler, 2019), often leading to sterility and unproductiveness of the host plant (Namba et al., 2019).

Plants react to phytoplasma invasion by mechanical occlusion of sieve pores, which is due to a fast plugging by specialized proteins (Will and van Bel, 2006; Furch et al., 2007; Pagliari et al., 2017), followed by a slower constriction due to additional deposition of callose around the pores (Musetti et al., 2010; Santi et al., 2013a; Musetti et al., 2013). In Arabidopsis thaliana, 12 genes (CALS1-12) encoding for callose synthase enzymes have been found (Xie and Hong, 2011). The diverse CALS-gene expression patterns throughout the plant are indicative of different local roles (Ellinger and Voigt, 2014). As for the phloem, Barratt et al., (2011) and Xie et al., (2011) demonstrated that the AtCALS7 gene encodes for Callose Synthase 7 (AtCALS7), the enzyme responsible for callose deposition around sieve pores. AtCALS7 also regulates sieve-plate development during phloem differentiation, which is an important determinant of mass flow in mature sieve tubes, and, hence, influences sink carbohydrate availability and plant growth (Xie et al., 2011).

Synthesis of the cell-wall polymer callose (1,3- $\beta-D$-glucan) requires sucrose units that are cleaved by sucrose synthases into fructose and UDP-glucose, the substrate for callose synthase. Arabidopsis callose synthase proteins contain multiple transmembrane domains that are clustered into two regions (N-terminal and $\mathrm{C}$-terminal), leaving a large hydrophilic central loop that faces the cytoplasm. This loop contains the putative catalytic domain, which has been further subdivided into two domains: the UDP-glucose binding domain and the glycosyltransferase domain (Chen and Kim, 2009). In the sieve tubes of Arabidopsis, two membrane-bound sucrose synthase isozymes, AtSUS5 and AtSUS6 (Barratt et al., 2009), associated with AtCALS7 form a unique enzyme complex (Bieniawska et al., 2007; Ruan, 2014; Stein and Granot, 2019).

Sucrose is of paramount importance in plant-pathogen interactions (Bolton et al., 2009; Rojas et al., 2014; Fatima and Senthil-Kumar, 2015; Dodds and Lagudah, 2016; Lee et al., 2016). Sugars are involved in several signaling processes (Lecourieux et al., 2014) and contribute to the plant immune response, as priming molecules for rapid activation of defense against biotic and abiotic stresses (Bolouri-Moghaddam and van Den Ende, 2012). Moreover, sucrose is the ideal carbon source owing to its high concentration in SEs (van Bel and Musetti, 2019). As result, sucrose host metabolism is often affected by 
100 phytoplasmas (Santi et al., 2013b; De Marco et al., 2016; Prezelj et al., 2016) given their

101 full dependence on the host (Oshima et al., 2013; Musetti et al., 2016).

102 Assumed the intricate and interdependent roles of callose and sugars in phytoplasma

103 infection and the functional connection between callose synthase and sucrose synthases

104 (Bieniawska et al., 2007; Ruan, 2014; Santi et al., 2013a; Stein and Granot, 2019) we

105 investigated the consequences of phytoplasma infection and the role of CALS7 in sucrose

106 metabolism and cell-cell transport of carbohydrates. Wild-type and Atcals7ko [a mutant

107 line in which the gene encoding for AtCALS7 was silenced (Barratt et al., 2011)]

108 Arabidopsis plants, healthy or infected with Chrysanthemum Yellows (CY)-phytoplasma,

109 were compared regarding plant morphology, sieve-tube substructure, gene expression

110 and carbohydrate amount. We investigated whether the loss of AtCALS7 in CY-infected

111 Arabidopsis would affect plant growth, symptom expression and phytoplasma titre.

112 Furthermore, we evaluated whether the loss of AtCALS7 had an impact on: i) the SE

113 ultrastructure; ii) the distribution of callose deposits at the plasmodesmata in midrib cortical

114 parenchyma and in leaf epidermis; iii) the amount of sucrose, glucose, fructose,

115 myoinositol, sorbitol, arabinose and raffinose in midrib tissues; iv) the expression patterns

116 in midrib tissues of several genes involved in sugar transport and metabolism.

117 The results indicate that, apart from the negative role in the sieve-plate occlusion, loss of

118 AtCAL7 appears to confer increased susceptibility to CY-phytoplasma infection, due to

119 alterations in symplasmic connectivity and expression of genes involved in sugar

120 metabolism and membrane transport in Atcals7ko plants. 


\section{Results}

124

125

126

127

128

129

130

131

132

133

134

135

136

137

138

139

140

141

142

143

144

145

146

147

148

149

150

151

152

153

154

\section{Phloem translocation speed}

The speed of phloem translocation was measured in healthy wild-type and Atcals7ko Arabidopsis plants (Fig. 1) through the label with $\mathrm{C}^{14}$. The linear translocation velocity (expressed as $\mathrm{cm} \mathrm{h}^{-1}$ ) was approximatively $50 \%$ lower in Atcals7ko mutants than in wildtype plants. The average speed in wild-type plants was $10.2 \pm 1.6 \mathrm{~cm} \mathrm{~h}^{-1}$, while it was $5.0 \pm$ $2.0 \mathrm{~cm} \mathrm{~h}^{-1}$ in mutants. As the infected plants developed very short stems (wild-type) or failed to do so (Atcals7ko, Fig. 2C), it was not possible to determine the translocation speed in CY-infected plants.

\section{Phenotypes of healthy and CY-infected Arabidopsis lines and phytoplasma titre}

Symptom appearance and rosette growth and weight (Fig. 2A and B) of wild-type and Atcals7ko plants, healthy or infected by CY-phytoplasmas were compared. Floral stem length was also evaluated in plants grown under long-day light conditions (Fig. 2C). Moreover, phytoplasma titre was quantified in infected Arabidopsis lines by qPCR (Fig. $2 \mathrm{E})$.

Atcals7ko plants were smaller than wild-type plants, having stunted growth (Fig. 2A) and leaves with a thick main vein. Fresh weight of rosettes (Fig. 2B) was reduced in Atcals7ko plants (average weight: $4.8 \pm 1.5 \mathrm{~g}$ ) to the $56 \%$ as compared to the wild-type plants (average weight: $8.5 \pm 1.2 \mathrm{~g}$ ). Following CY-infection, rosette fresh weight, on average, was affected by a reduction of $16 \%$ in wild-type plants $(7.1 \pm 1.0 \mathrm{~g})$ and $27 \%$ in the Atcals7ko line $(3.5 \pm 2.2 \mathrm{~g})$ in comparison with their respective healthy controls. In both lines, leaves that emerged after phytoplasma inoculation were yellowish, small, narrowed, with a thicker main vein and a shorter petiole. When grown under long-day light conditions, the mutant line developed a floral stalk reduced to the $41 \%$ in length (average length: 16.3 $\pm 0.7 \mathrm{~cm}$ ) in comparison to the wild-type (average length $26.8 \pm 2.5 \mathrm{~cm}$ ) (Fig. $2 \mathrm{C}$ and D). CY-infected wild-type plants produced a stalk reduced by $88 \%$ (average length $3.3 \pm 3.2$ $\mathrm{cm}$ ) compared to the healthy controls, while CY-infected Atcals7ko mutants failed to produce a stalk at all (Fig. 2C and D). Although not significantly different, phytoplasma titre resulted, on average, higher in mutant plants (i.e. 4.4E+08 phytoplasma GUs in $100 \mathrm{mg}$ of leaf sample) than in the wild-type (i. e. $1.8 \mathrm{E}+08$ phytoplasma GUs in $100 \mathrm{mg}$ of leaf sample) (Fig. 2E).

\section{Electron-microscopic observations on midrib vascular bundles}



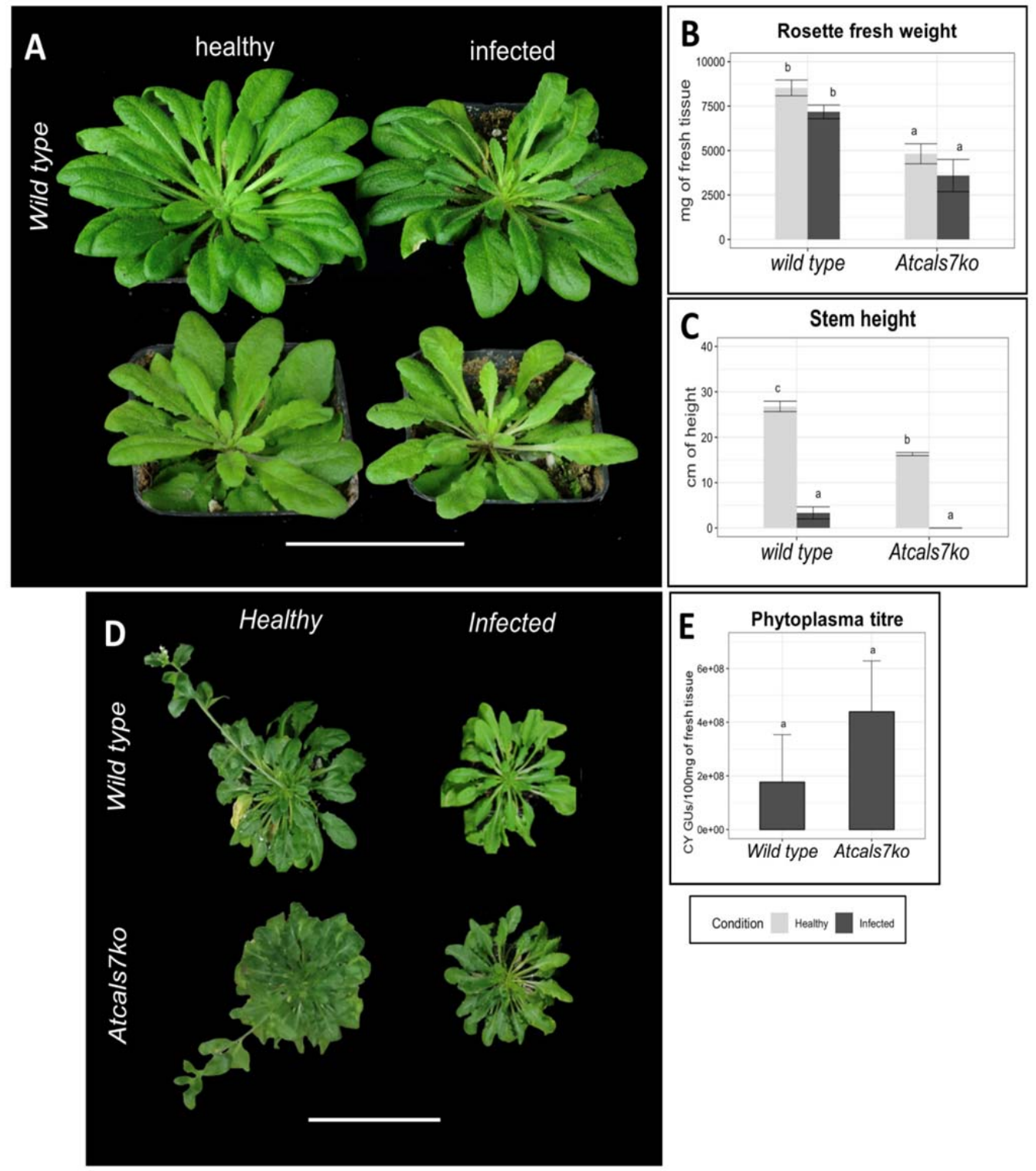

Fig. 2. Plant phenotype and phytoplasma titre in wild-type and Atcals7ko line. Representative images of healthy and CY-infected wild-type and Atcals7ko plants. Following CY-infection, at 20 days after the inoculation access period (IAP), both plant groups showed yellowish small leaves. Leaves emerged after phytoplasma inoculation were shorter, with thicker main vein and smaller petiole (A). Regardless phytoplasma infection, rosette fresh weight is significantly reduced in Atcals7ko plants in with wild-type (B). Although not significantly different, phytoplasma titre increases in the mutant line in comparison to the wild type (C). In 60-day-old Arabidopsis plants, grown under long day conditions, stem was well developed in healthy wild-type but reduced in length in Atcals7ko plants (D, E). Stem length resulted strongly reduced in infected wild-type individuals and absent in the Atcals7ko mutants (D, E).

Different letters indicate different means according to the non-parametric Kruskal-Wallis post hoc test, $\mathrm{P}<0.05$. Error bars indicate Standard Error of the Mean of 8 biological replicates for each condition. Plant weight (C) 20 days IAP expressed as $\mathrm{mg}$ of fresh tissue. Statistical analysis was performed using the Tukey HSD test as the post hoc test in a two-way ANOVA. Different letters $(a, b)$ above the bars indicate significant differences, with $P<0.05$. Error bars indicate the Standard Error of the Mean of 8 bcomparisoniological replicates for each condition. 


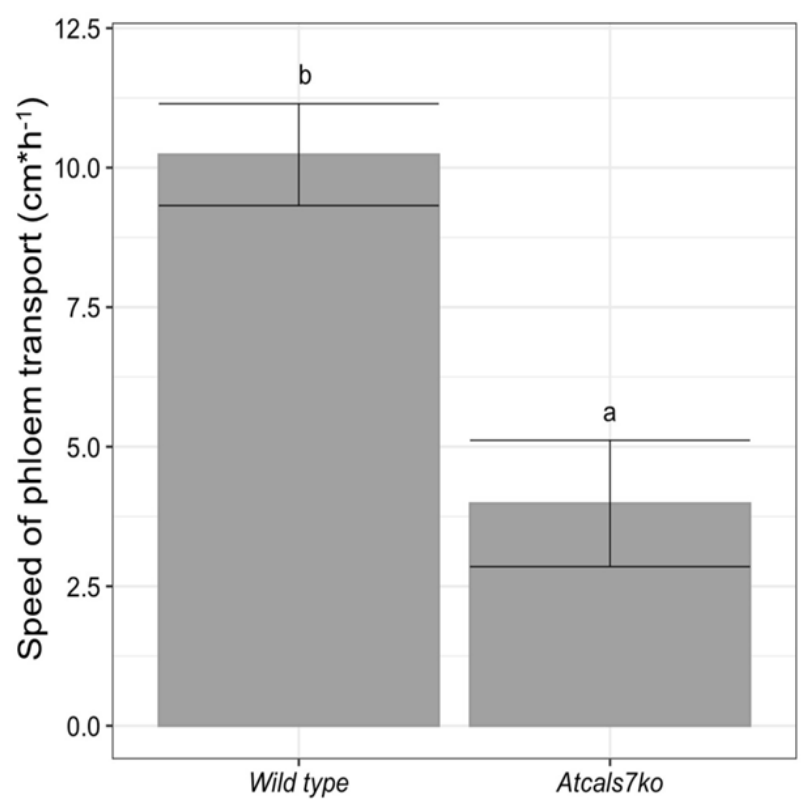

Fig. 1. Phloem transport speed in wild-type and Atcals7ko Arabidopsis lines.

Carbohydrate translocation speed along the phloem, measured with ${ }^{14} \mathrm{C}$ isotope. The speed is expressed as $\mathrm{cm} / \mathrm{h}$ and it is calculated by average time of arrival of the ${ }^{14} \mathrm{C}$ label in the stem phloem tissue near the $\mathrm{x}$-ray detector. Statistical analysis was performed using the Tukey HSD test as the post hoc test in a twoway ANOVA. Different letters $(a, b)$ above the bars indicate significant differences, with $\mathrm{P}<$ 0.05. Error bars indicate the Standard Error of the Mean of 4 biological replicates for each condition. 
bioRxiv preprint doi: https://doi.org/10.1101/2021.06.25.449948; this version posted June 26, 2021. The copyright holder for this preprint (which was not certified by peer review) is the author/funder, who has granted bioRxiv a license to display the preprint in perpetuity. It is made available under aCC-BY-NC-ND 4.0 International license.

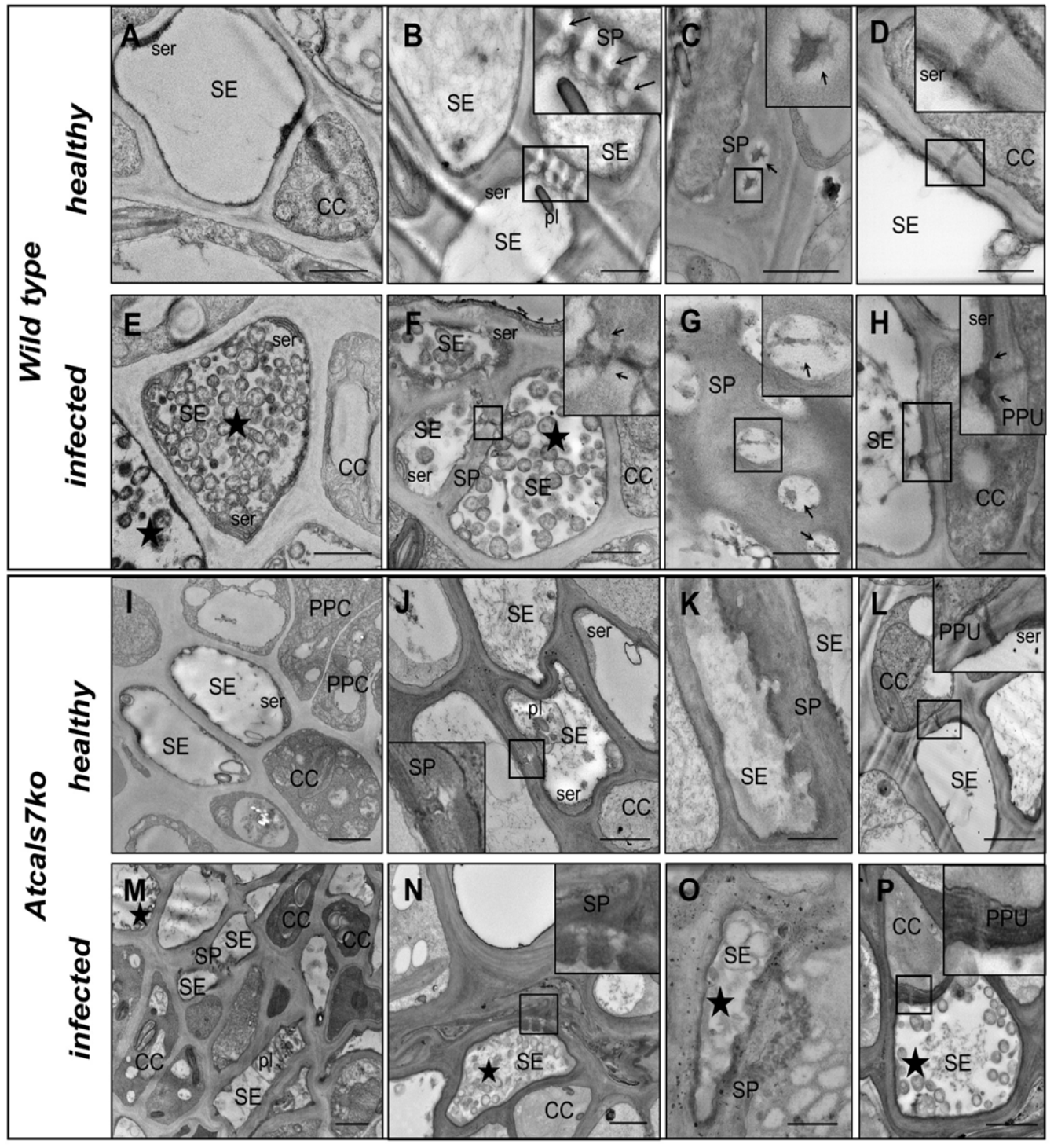

Fig. 3. Representative TEM micrographs of the sieve elements of healthy and CY-infected Arabidopsis lines. A-H. Cross-sections of midribs from healthy (A-D) and infected (E-H) wild-type Arabidopsis leaves. Healthy samples present unaltered sieve elements and companion cells (A), with a regular shape and no signs of necrosis or subcellular aberrations. The sieve pores, at both lateral (B) and ordinary (C) sieve plates, are not constricted by callose collars, pore-plasmodesma units are open and show their typical branched shape with sieve endoplasmic reticulum located in the proximity of the orifice (D). In infected midribs, numerous phytoplasmas are visible inside the sieve elements $(E)$ and at the sieve plate $(\mathbf{F})$. Lateral sieve plates have sieve pores mostly open $(\mathbf{F})$, whereas in ordinary sieve plates (G) pores are constricted by callose depositions. Pore-plasmodesma units display a similar morphology as in healthy samples, with a thin callose line at the sieve-element side (H). I-P. Crosssections of midribs from healthy (I-L) and CY-infected (M-P) Atcals7ko Arabidopsis leaves. In healthy Atcals7ko samples, phloem cells are apparently well structured (I), but the sieve plates show aberrant morphology (J, K). Pores lack callose and appear not developed (J, K). Pore-plasmodesma units are branched, similar as in wild-type samples (L). In CY-infected Atcals7ko samples, many phloem cells evidence thick cell walls $(\mathbf{M})$, others are collapsed $(\mathbf{N})$. Sieve plates are deformed, thickened $(\mathbf{N}, \mathbf{0})$ and pores are filled by electron-opaque material $(\mathbf{N})$. Pore-plasmodesma units appeared large, without welldefined branches $(\mathbf{P})$.

Arrows: callose; CC: companion cell; PPC: phloem parenchyma cell; pl: plastid; PPU: poreplasmodesma unit; SE: sieve element; ser: sieve element reticulum; SP: sieve plate; star: phytoplasmas. Bars correspond to $1 \mu \mathrm{m}$. 
1585 different plants of both lines were analysed. Healthy wild-type samples showed a regular

159 SE and CC ultrastructure (Fig. 3A-D). In lateral (Fig. 3B) and transverse (Fig. 3C) sieve

160 plates, sieve pores were surrounded by a callose lining (Fig. 3B and C) that did not

161 constrict their lumen. At the SE/CC wall interface, sieve endoplasmic reticulum (SER) was

162 visible in front of the open, typically branched pore-plasmodesma units (PPUs, Fig. 3D). In

163 infected wild-type midribs (Fig. 3E-H), numerous phytoplasmas were visible in the SE

164 lumen (Fig. 3E) or in proximity of the sieve plates (Fig. 3F). In transverse sieve plates (Fig.

$1653 \mathrm{G}$ ), the pores were constricted by callose deposition (Fig. 3G), whereas they appeared

166 mostly open in lateral sieve plates (Fig. 3F). PPUs were open and displayed a slightly

167 thickened callose lining at the SE side (Fig. 3H). As in healthy plants, SER was observed

168 near the PPU opening in infected plants (Fig. $3 \mathrm{H})$.

169 In the healthy Atcals7ko line (Fig. 3I-L), SEs and CCs appeared identical to those in 170 the wild-type (Fig. 3I). Sieve-element protein filaments, plastids and SER were readily 171 recognizable in the SE lumen or close to the plasma membrane (Fig. 3J). In accordance 172 with previous studies (Barratt et al., 2011; Xie et al., 2011), however, sieve plates lacked 173 callose and showed an aberrant morphology (Fig. 3J and K). Some sieve-pore channels 174 seemed to be incompletely open or not fully developed (Fig. 3J and K), whereas PPUs 175 displayed a normal, branched appearance (Fig. 3L). Inside SEs of the infected Atcals7ko 176 line phytoplasmas were visible (Fig. 3M-P). Many SEs possessed thick walls (Fig. 3M), 177 while others had collapsed (Fig. 3N). Like in healthy samples, sieve plates appeared to be 178 damaged, with sieve pores filled by electron-opaque material (Fig. 30). PPUs were 179 enlarged without a well-defined branching (Fig. 3P). In the proximity of PPUs, SER was recognizable in the SEs of both healthy and CY-infected plants (Fig. 3L and P).

181 Confocal laser scanning microscopy analyses and imaging

182 Confocal laser scanning microscopy (CLSM) in combination with aniline blue staining were used to evaluate presence, number and distribution of blue-fluorescent callose 184 deposits, the fluorescence intensity and the integrated density (i.e. the sum of intensity within the area of the region of interest) in various leaf tissues of wild-type and Atcals7ko

186 plants, healthy or CY-infected. Fluorescent regions represented the callose lining along 187 the symplasmic corridors of SE/CC complexes (Fig. 4); the discrete fluorescent dots in 188 midrib cortical parenchyma (Fig. 4) and epidermis (Fig. 5) localized callose collars around 189 plasmodesmata. In infected wild-type samples, intense aniline blue signals revealed a 190 significant increase in callose deposits at the SE level (Fig. 4B), in comparison to their 
bioRxiv preprint doi: https://doi.org/10.1101/2021.06.25.449948; this version posted June 26, 2021. The copyright holder for this preprint (which was not certified by peer review) is the author/funder, who has granted bioRxiv a license to display the preprint in perpetuity. It is made available under aCC-BY-NC-ND 4.0 International license.
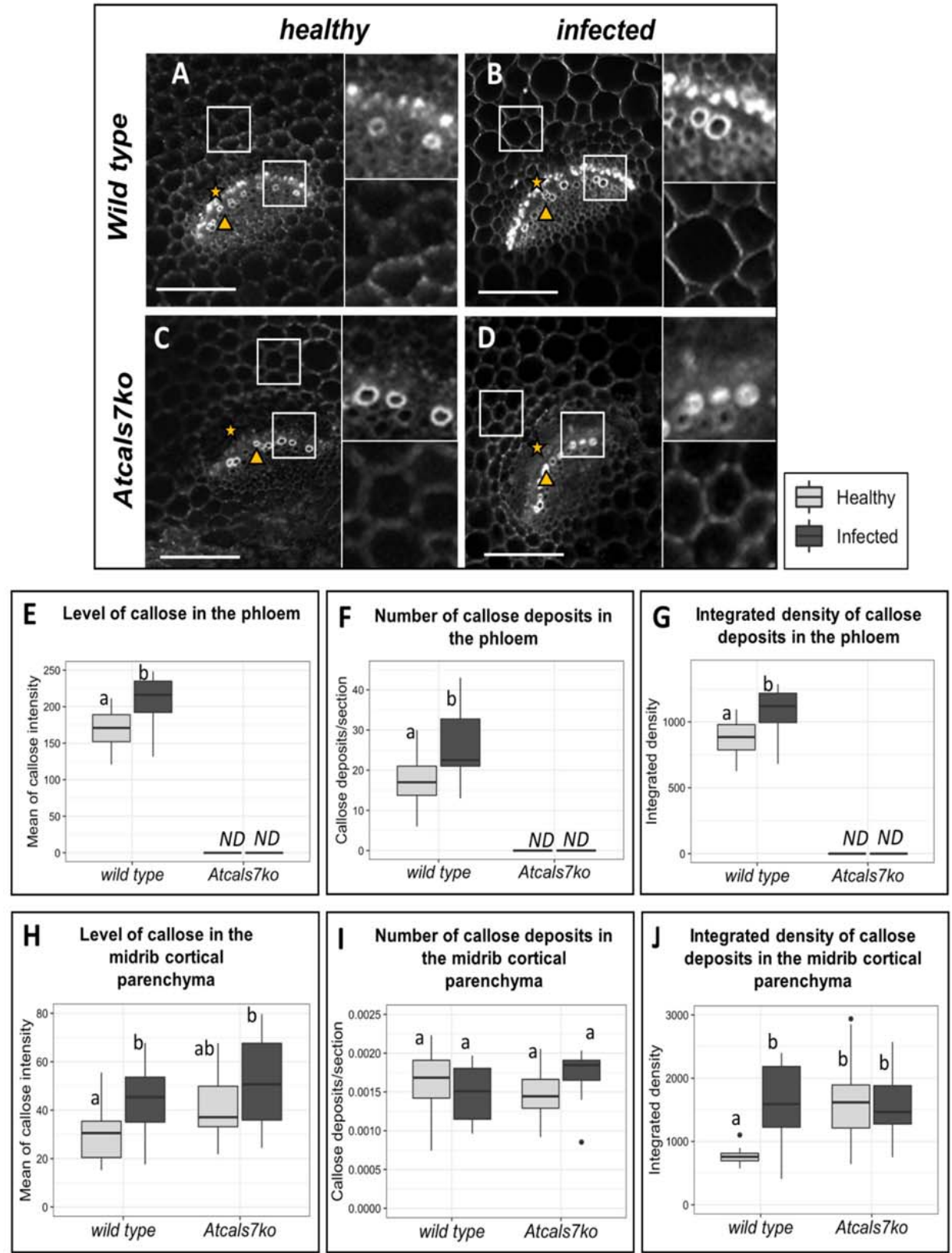

Fig. 4. Single-plane confocal micrographs and imaging of callose deposits at plasmodesmata in phloem area and in parenchyma of healthy and $\mathrm{CY}$-infected Arabidopsis midribs. Fluorescent spots indicate callose depositions in the phloem area of healthy (A) and CY-infected (B) wild-type samples. The fluorescence intensity $(\mathbf{E})$, the number $(\mathbf{F})$ and the integrated density [i.e. the sum of the intensity in the region of interest (ROI)] of the deposits (G) is significantly higher in the infected midribs compared to the healthy ones. No signal is visible in Atcals7ko midribs (C, D). Punctate dots, indicating callose deposits at plasmodesmata are visible in the midrib parenchyma of all samples (A-D). In the ROI, fluorescence intensity $(\mathbf{H})$ and number of dots $(\mathbf{I})$ are not different in healthy samples, but the integrated density (i.e. the sum of the intensity within the area of the region of interest, ROI) is significantly higher in the mutant line than in the wild-type $(\mathrm{J})$. Following infection, the fluorescence intensity and the integrated density of the signal in the ROI significantly increased only in wild-type samples. In the pictures, stars indicate phloem and triangle indicate xylem. Statistical analysis was performed using the Tukey HSD test as the post hoc test in a two-way ANOVA. Different letters (a, b, c, d) above the bars indicate significant differences, with $P<0.05$. Boxplots were obtained from three biological replicates for each condition and almost 10 technical replicates for each sample. Bars correspond to $100 \mu \mathrm{m}$. 

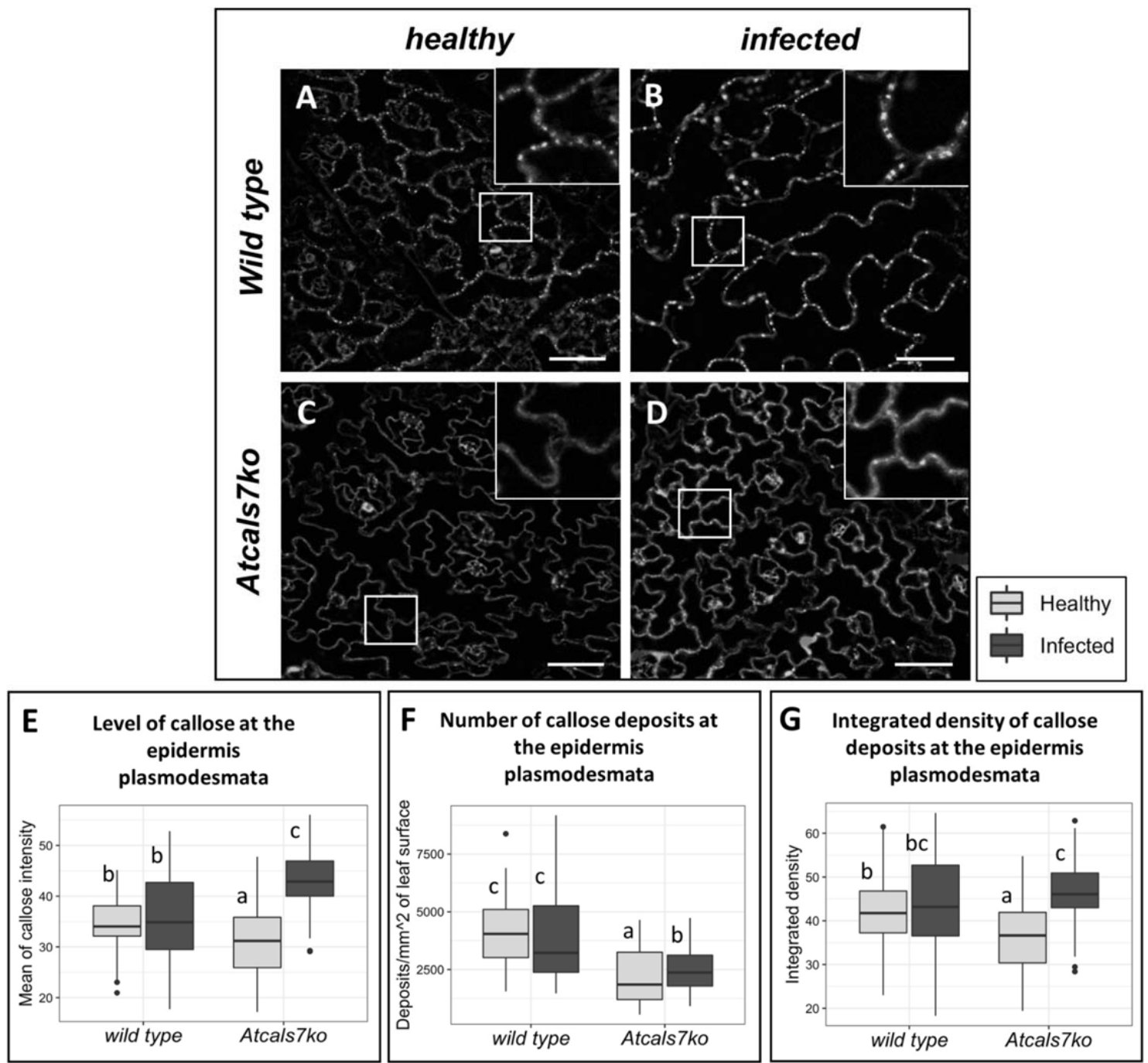

Fig. 5. Single-plane confocal micrographs and imaging of callose deposits at plasmodesmata in epidermal cells. Aniline blue fluorescent dots (A-D), their intensity $(E)$, number $(\mathbf{F})$ and integrated density (i.e. the sum of the intensity within the area of the region of interest, ROI, G) were assayed at leaf epidermis level in healthy and infected samples of both lines. In healthy samples, the intensity $(E)$ and the number of fluorescent dots (F), and the integrated density $(\mathbf{G})$ are lower in Atcals 7 ko line compared to the wild-type. Following CY-infection the three parameters significantly increased only in Atcals7ko line. Statistical analysis was performed using the Tukey HSD test as the post hoc test in a two-way ANOVA. Different letters $(a, b, c)$ above the bars indicate significant differences, with $P<0.05$. Boxplots were obtained from three biological replicates for each condition and almost 10 technical replicates for each sample. Boxplots were obtained from 5 biological replicates for each condition. Bar corresponds to $50 \mu \mathrm{m}$.

192 4F) and the integrated density (i.e. the sum of intensity within the area of the region of 193 interest) of deposits (Fig. 4G) were measured and compared in healthy and infected wild194 type samples. Infection induced a significant increase in callose deposition in SEs of wild195 type plants (Fig. 4E-G). As expected, no fluorescence was detected in the corresponding SE area in Atcals7ko samples, even in case of phytoplasma infection (Fig. 4C and D). 
Image analysis allowed us to quantify the fluorescent signals (plasmodesma-located dots) in cell walls of midrib cortical parenchyma (Fig. 4). In healthy samples of both Arabidopsis lines, the signal intensity (Fig. 4H) and number of fluorescent dots (Fig. 4I) did not differ significantly in the selected areas. However, the integrated density was significantly higher in mutant line than in wild-type plants. Following infection, the level of fluorescence and the integrated density significantly increased only in wild-type samples and not in the Atcals7ko line (Fig. 4H-J). In contrast to the situation in the cortical parenchyma, the intensity (Fig. 5E), the number of fluorescent dots (Fig. 5F), and the integrated density (Fig. 5G) in the epidermis of healthy plants were lower in Atcals7ko than in wild-type samples. Following CY-infection, the three parameters investigated increased significantly only in Atcals7ko line. In particular, the signal intensity in the infected mutant line was the highest as compared to the other samples (Fig. 5E), whereas the integrated density was similar to that found in infected wild-type samples (Fig. 5G).

With exception of autofluorescence of the xylem and chloroplasts no fluorescence signal was detected in unstained samples (Supplementary file 1).

\section{Sugar quantification in midrib tissues}

Sucrose, glucose, fructose, myoinositol, sorbitol, arabinose and raffinose were quantified in the midribs of wild-type and Atcals7ko plants (Fig. 6). No significant the controls in the wild-type line (Fig. 6A-G). In the Atcals7ko line, sucrose, glucose and myoinositol significantly increased in response to CY-infection (Fig. 6A, B and D). In particular, comparing with the amounts found in the midribs of healthy Atcals7ko plants, sucrose increased around 5-fold in infected Atcals7ko plants (Fig. 6A), glucose around 4-

221 fold (Fig. 6B), myoinositol around 2-fold (Fig. 6D).

\section{Gene expression analyses}

The expression level of the sieve-element specific callose synthase 7 gene (AtCALS7) was analyzed in midribs of healthy and CY-infected wild-type plants and was significantly up-regulated (around 2.5-fold) in infected plants (Fig. 7A). Moreover, the expression of genes involved in sugar metabolism and transport, in the midribs of source 

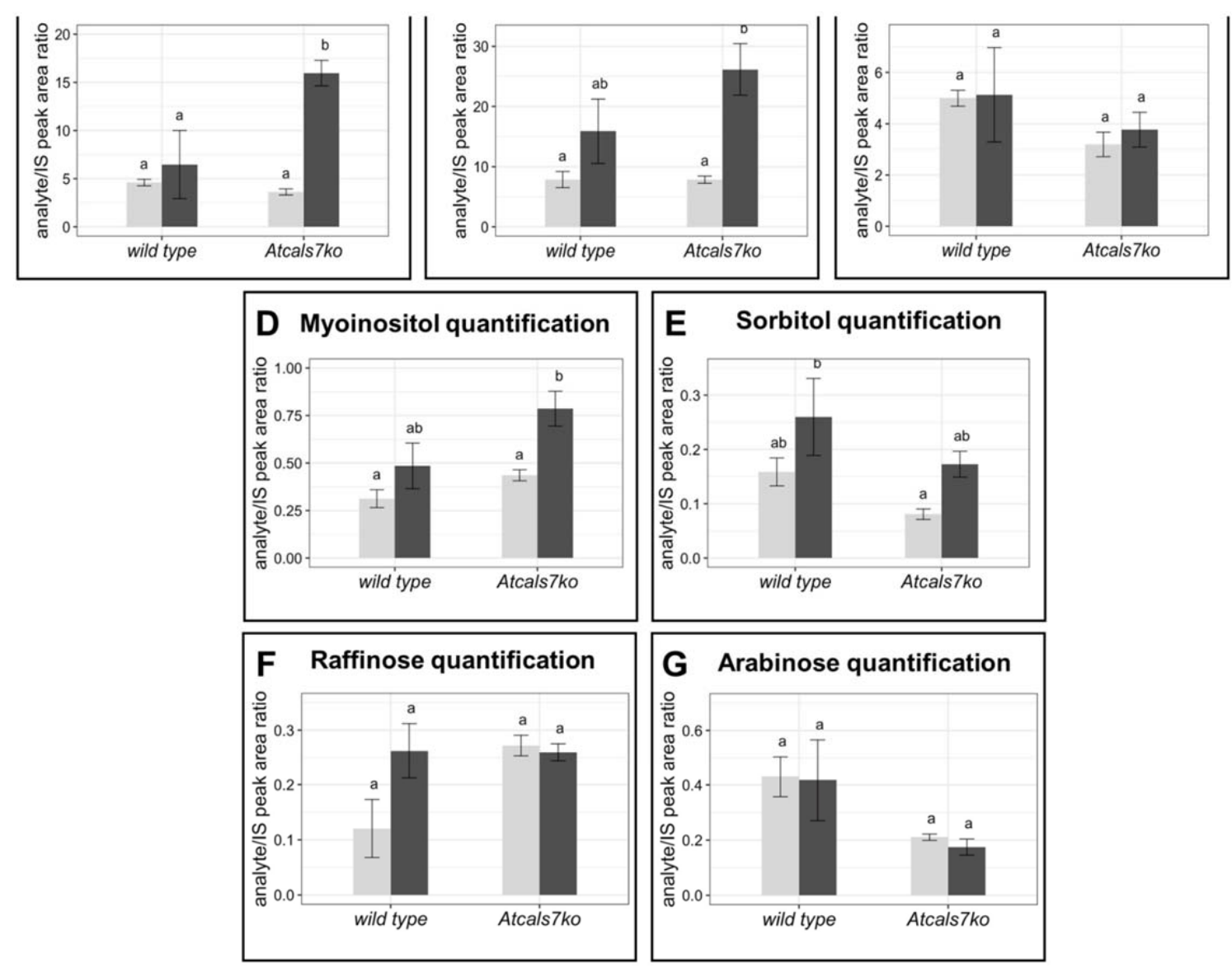

Fig. 6. Sugar quantification in the midribs of healthy and infected Arabidopsis lines. Sucrose (A), glucose (B), fructose (C), myoinositol (D), sorbitol (E), raffinose (F) and arabarabinose (G) were quantified in midribs of the two different lines, healthy or CY-infected. Data obtained were expressed as analyte/IS peak area ratio. Statistical analysis was performed using the Tukey HSD test as the post hoc test in a twoway ANOVA. Different letters $(a, b)$ above the bars indicate significant differences, with $\mathrm{P}<0.05$. Error bars indicate the Standard Error of the Mean of 4 biological replicates for each condition run in triplicate.

facilitators (AtSWEET11, AtSWEET12), cell wall invertases (AtCWINV1, AtCWINV6) were determined in the four plant groups under investigation (Fig. 8B-F).

AtSUS5 and AtSUS6 encode two sucrose synthases located in the SEs, which provide UDP-glucose as substrate for AtCALS7. Comparing the expression levels found in 233 healthy samples (i.e. wild-type versus Atcals7ko), AtSUS6 was significantly up-regulated in 234 the mutant line (Fig. 7B). AtSUS5 showed low expression levels which did not differ in the 
bioRxiv preprint doi: https://doi.org/10.1101/2021.06.25.449948; this version posted June 26, 2021. The copyright holder for this preprint (which was not certified by peer review) is the author/funder, who has granted bioRxiv a license to display the preprint in perpetuity. It is made available under aCC-BY-NC-ND 4.0 International license.

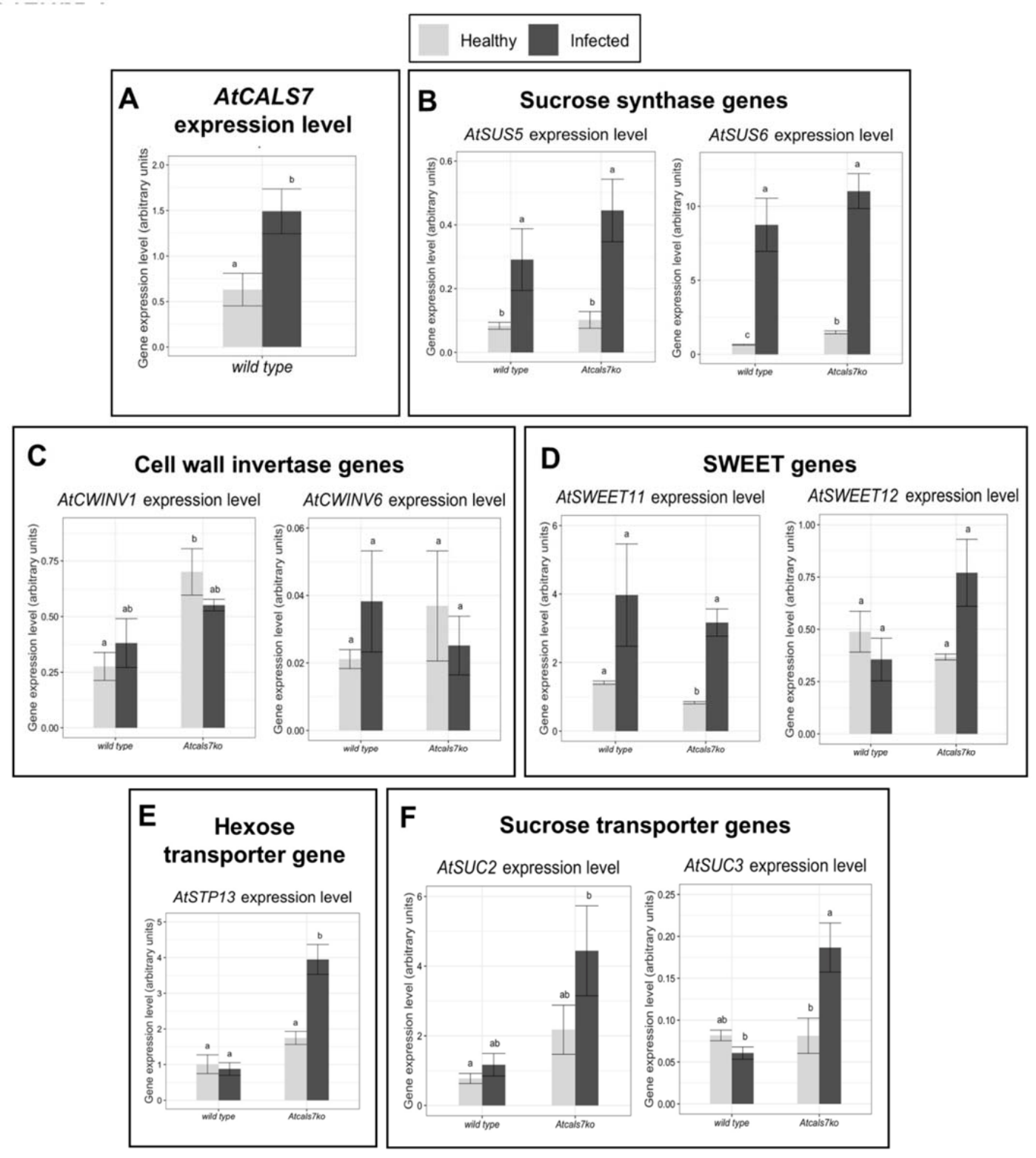

Fig. 7. Transcript profiling of genes involved in phloem callose synthesis and sugar transport and metabolism. A. Expression level of phloem callose synthase gene (AtCALS7) in healthy and CY-infected wild-type plants. B-F. Transcript profiling of sucrose synthases AtSUS5 and AtSUS6 (B), sucrose transporters AtSUC2 and AtSUC3 (C), SWEET sugar facilitators AtSWEET11 and AtSWEET12 (D), cell wall invertases AtCWINV1 and AtCWINV6 (E), the hexose transporter AtSTP13 (F). Healthy and infected plants belonging to the two lines were compared. Expression values were normalized to the UBC9 transcript level, arbitrarily fixed at 100, then expressed as mean normalized expression \pm SD (transcript abundance). Statistical analysis was performed using the Tukey HSD test as the post hoc test in a twoway ANOVA. Different letters ( $a, b, c, d)$ above the bars indicate significant differences, with $P<0.05$. Error bars indicate the Standard Error of the Mean of 5 biological replicates for each condition run in triplicate.

235 two lines. Following CY-infection, AtSUS5 transcripts increased 3.5 and 5 times in wild236 type and mutant lines, respectively, while AtSUS6 transcripts increased 13.5 and 7.5 times

237 (Fig. 7B). AtCWINV1 and AtCWINV6 encode two cell wall invertases, involved in the 

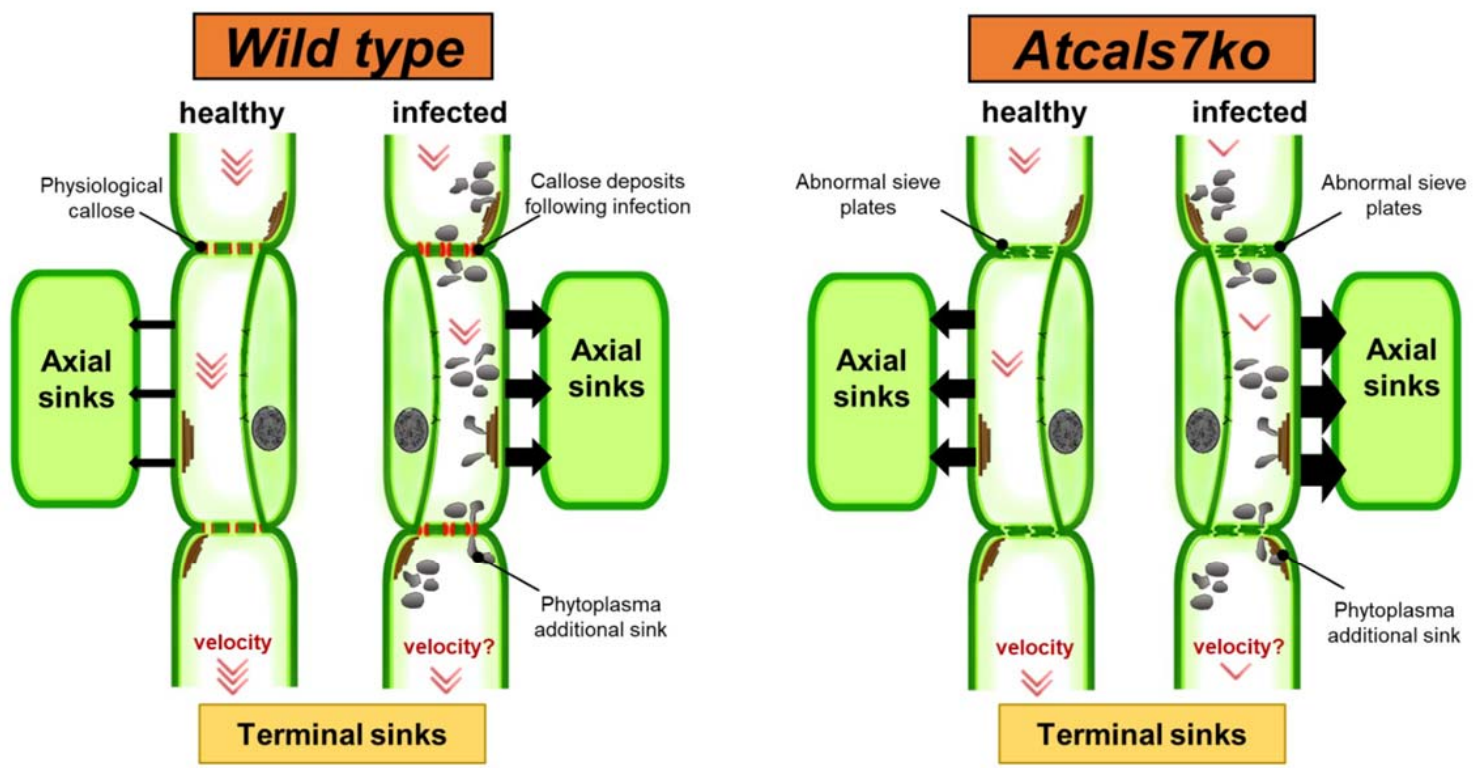

Fig. 8. Hypothetical model for the impact of functional (wild-type line) or aberrant (Atcals7ko line) sieve plates on the photoassimilate investment into terminal or axial sinks. The red arrowheads quantify the extent of investment in terminal sinks, which is correlated to the translocation speed. The size of the horizontal black arrows quantifies photoassimilates which are invested in axial sinks. Photoassimilate investment into terminal sinks is lower in the Atcals7ko line, due to aberrant sieve plates, which results in more escape of carbohydrates along the pathway towards the axial sinks. In case of phytoplasma infection, photoassimilate investment into terminal sinks could be more affected, favoring not only the axial sink proliferation, but also the phytoplasma (additional sink) nourishment.

irreversible cleavage of sucrose into glucose and fructose in the apoplast. Cell wall invertases represent a path for hexose production, as an alternative to sucrose synthases.

240 The expression level of AtCWINV1 was significantly higher in the midrib of healthy 241 Atcals7ko plants (Fig. 7C) than in the other plant groups. AtCWINV6 showed a similar 242 trend, but the very low expression level prevented us from calculating a significant 243 difference between the lines (Fig. 7C). The gene encoding the sugar efflux facilitator 244 AtSWEET11 was down-regulated in healthy Atcals7ko midribs in comparison with the 245 healthy wild-type (Fig. 7D). In response to infection, it was significantly over-expressed in 246 Atcals7ko line (Fig. 7D). Transcription levels of AtSWEET12 were unchanged in all tested 247 samples (Fig. 7D). The expression level of AtSTP13, a gene encoding a vascular hexose 248 transporter, was similar in the midribs of both lines, but was enhanced significantly (around 249 5.5-fold) in the infected Atcals7ko line (Fig. 7E). The transcripts of the two genes AtSUC2 250 and AtSUC3, encoding sucrose transporters located respectively in CCs and in SEs 251 (Meyer et al., 2004) were analyzed in the midribs of both lines (Fig. 7F). The expression 252 level of AtSUC2 did not significantly change in all plant groups, while AtSUC3 was 253 significantly up-regulated only in the Atcals7ko line following CY infection (Fig. 7F). 
254 All in all, three general trends were visible regarding the gene expression in infected 255 mutant and wild-type plants in comparison with their healthy controls. Modulation was 256 absent for AtCWINV1 and AtCWINV6 in both lines, AtSUS5 and AtSUS6 were upregulated 257 in both lines, while AtSWEET11, AtSUC3, and AtSTP13 were upregulated in mutants only. 


\section{Discussion}

Early reports already noted that sieve pores are rich in callose (Eschrich, 1956), which is produced by transmembrane callose synthases, a gene family that comprises 12 members in Arabidopsis and is responsible for cell- or tissue-type specific callose production (Verma and Hong, 2001). Barratt et al., (2011) and Xie et al., (2011) demonstrated that, in Arabidopsis thaliana, callose deposition at the sieve plates is regulated by a callose synthase gene, AtCALS7, which is spatially associated with the phloem-specific sucrose synthase genes (AtSUS) 5 and 6 (Barratt et al., 2009). The same authors attributed a role to both sucrose synthases in providing the UDP-glucose for callose synthesis around sieve pores. Callose synthesis requires several steps, including the glucosyl-group transfer by a transferase (Hong et al., 2001; Bonke et al., 2003; Barratt et al., 2009), which renders credibility to a callose synthase complex, composed of diverse proteins (Schneider et al., 2016).

For plants infected by phytoplasmas, increased callose deposition at the sieve plates has been described since the '70s (Braun and Sinclair, 1978) as a means of constricting sieve pores and limiting pathogen spread (Musetti et al., 2013). Enhanced callose deposition was associated with an upregulation of callose synthase genes in phytoplasmainfected apple tree, grapevine and tomato (Musetti et al., 2010; Santi et al., 2013b; De Marco et al., 2016). The structural and physiological modifications were seemingly consistent with transcriptional changes in sucrose transport and metabolism following infection (Santi et al., 2013b), and appeared worth investigating. As phytoplasmas are phloem-limited pathogens (van Bel and Musetti, 2019), we used healthy and phytoplasmainfected Arabidopsis lines, wild-type or Atcals7 knock-out (Barratt et al., 2011; Xie et al., 2011) to investigate whether and to what extent the sieve-element specific callose synthase 7 (AtCALS7) interferes with plant sugar translocation and metabolism in response to phytoplasma infection.

\section{Lack of AtCALS7 affects carbohydrate translocation speed and plant growth}

Formulae for mass flow determination (De Schepper et al., 2013 and literature therein) predict that the shape of sieve plates controls the translocation velocity. We measured the speed of carbohydrate transport in wild-type and Atcals7ko Arabidopsis plants using a non-invasive method (Vincent et al., 2019). As expected, Atcals7ko mutants display a decreased translocation speed consistent with the distorted shape of the sieve 
292 pores (Figs. 3J and K and Barratt et al., 2011). Reduction of the linear translocation speed 293 seems to have an effect on the carbohydrate supply of the sinks. As a result, growth is 294 slowed down as expressed by the reduction of rosette fresh weight and stem length in mutant plants (Fig. 2B and C).

296

297

298

299

300

301

302

303

304

305

306

307

308

309

310

311

312

313

314

315

316

317

318

319

320

321

322

323

324

The decreased growth indicates that the balance between the supply of terminal and axial sinks is appreciably disturbed in mutants (e.g. Hafke et al., 2005). Since sugar concentrations and expression levels of sugar-handling enzymes are similar in wild-type and mutant plants (Figs. 6 and 7), the disturbance of nutrient distribution is likely due to mechanical alterations. At the same phloem-loading rates, the concentration of soluble carbohydrates must be higher in sieve-tube saps transported at lower velocities. At reduced velocities, moreover, solute retrieval rates by axial sinks along the transport pathway are expected to be higher due to the longer residence times of translocate in the transport pathway (Horwitz, 1958). Both factors effectuate an increased retrieval by axial sinks, even if their carbohydrate transporters are not upregulated (Fig. 8). And finally, a more efficient symplasmic connectivity in the cortex of mutants allows a quicker transfer to and storage by axial sinks (Fig. 4J). Knocking-out AtCALS7 thus restricts the nutrient supply of terminal sinks (Fig. 8), while the axial sinks seem to profit from the reduced translocation speed (Fig. 1).

Phytoplasma infection highlights another mode of AtCALS7 impact. In response to CY infection, mutants and wild-type plants showed respectively an additional $27 \%$ and $16 \%$ rosette-weight loss in comparison with their healthy controls. CY-infected plants were incapable of developing normal stems (Fig. 2C and D), with mutant plants showing stronger effects. Even if the increased callose deposition did not constrict all sieve plates (Fig. 3F and G; Braun and Sinclair, 1978; Gallinger et al., 2021), stem stunting reported in CY-infected wild-type plants (Pagliari et al., 2016) is indicative of a strong disturbance of phloem functions imposed by phytoplasmas (Maust et al., 2003). The modified distribution patterns suggest that nutrients are invested in additional sinks in the form of phytoplasmas at the cost of the terminal sinks (Fig. 8). The investment in phytoplasma growth would be in the mutant plants twice as high as in the wild-type plants. As a matter of fact, the phytoplasma titre is indeed about twice as high in the mutants (Fig. 2E). Phytoplasmas seem to exploit the absence of AtCALS7 for better recruitment of resources for their growth. Below, we will explore in the sections the underlying factors that explain the higher fitness of phytoplasmas in mutant plants. 


\section{The altered symplasmic communication between cortex cells in the Atcals7ko line} favors phytoplasma infection

Plasmodesmata and their gating mechanisms could be of importance for the survival, dissemination and effector dispersal of phytoplasmas (van Bel and Musetti, 2019 and literature therein). In this context, callose deposition that influences plasmodesmal functional dynamics, by modulating the passage of molecules through the cytoplasmic sleeve, is of paramount importance (Sager and Lee, 2018).

To check the callose distribution and deposition level in different tissues of wild-type and Atcals7ko Arabidopsis midribs, healthy or CY-infected, aniline-blue that becomes fluorescent by binding to callose, was used (Zavaliev and Epel, 2015). As expected, the fluorescent dots appeared in the SE/CC area in the midribs of wild-type plants (De Marco et al., 2016; Pagliari et al., 2016) but not in the Atcals7ko mutants. This is in agreement with the TEM results (Fig. 3; Barratt et al., 2011; Xie et al., 2011). In midrib cortical parenchyma of both Arabidopsis lines, aniline-blue staining showed callose deposition at the plasmodesmata, as numerous fluorescent punctate dots at the cell boundaries (Levy et al., 2007). In the two Arabidopsis lines, the number of deposits and the fluorescence intensity was not significantly different, in average, within the region of interest. Nevertheless, considering the integrated density of the dots in that area, this was significantly higher in the Atcals7ko line than in the wild-type, indicating differences in plasmodesmal functional dynamics. It has been recently suggested that callose could increase the elasticity rather than rigidity of the wall matrix (Abou-Saleh et al., 2018), favoring selective symplasmic transport. This is in line with a more efficient symplasmic connectivity in the cortex of mutants to promote axial sink nourishment, as suggested above.

Callose level was strongly enhanced in cortical tissues of infected wild-type plants, but not in the mutant (Fig. 4H). This lack of additional callose deposition suggests that the cortical plasmodesmata in mutants do not respond to phytoplasma infection. This may have two consequences in mutant plants. First, effectors may disseminate more effectively into extra-fascicular tissues (van Bel and Musetti, 2019) and second, hexose and sucrose move more easily from cell to cell.

In the epidermal tissue, the number of fluorescent dots, the signal intensity and the integrated density is lower in Atcals7ko samples compared to the wild-type, suggest an 
357 attempt of mutant plants to establish the symplasmic continuity in tissues far from the

358 phloem, to control symplasmic return of assimilates towards the region adjoining the 359 phloem. In case of phytoplasma infection, callose deposition at epidermis level increases 360 in the mutant line, which infers that plasmodesmata increase the capability to control 361 symplasmic molecular movement also in tissues not directly involved in pathogen 362 interaction.

\section{Phytoplasma infection increases sugar contents in midribs of Atcals7ko plants}

364

In midribs of healthy source leaves, sugar levels (i.e. sucrose, glucose, fructose, myoinositol, sorbitol, raffinose, arabinose) were similar in the wild-type and Atcals7ko Arabidopsis lines. The production of sucrose and its related catabolites is not influenced by the loss of AtCALS7, although the exact metabolite distribution per cell type is uncertain due to the use of entire midribs with diverse cell types.

An enhanced rate of carbohydrate metabolism in response to phytoplasma infection is derived from increased glucose, sucrose and myoinositol levels in midribs of mutant and wild-type plants (Santi et al., 2013a, 2013b; Yao et al., 2019). This upregulation of the soluble sugar level is more significant in mutants than in wild-type plants (Fig. 7). In the wild-type line, the significantly increased level of AtSUS5 and AtSUS6 transcripts (Fig. 7) is in line with the higher expression level of AtCALS7 (Fig. 7; Barratt et al., 2011), to provide precursors (i.e. UDP-glucose) for callose synthesis (Tan et al., 2015; De Marco et al., 2020), and for the production of carbon skeletons for synthesis of defense-related compounds (Bolouri-Moghaddam et al., 2010; Musetti et al., 2013). In Atcals7ko infected plants, increased photoassimilate investment into axial and additional sinks (such as phytoplasmas) would explain enhanced carbohydrate content and the up-regulation of AtSUS5 and AtSUS6 genes, which provide energy at the infection site (Yao et al., 2019; 2020). In the infected mutant line high amount of myoinositol was detected. In the absence of callose, myoinositol could be useful to modulate SE wall plasticity as an attempt to respond to the stress imposed by phytoplasmas, as demonstrated for other environmental cues (Wu et al., 2018). Myoinositol is indeed an excellent carbon source for cell wall constituents, such as pectin and hemicellulose (Loewus et al.,1962), which are, together with cellulose, the major types of polysaccharides in Arabidopsis cell walls (Bethke et al., 2016). 
Loss of AtCALS7 leads to concerted channeling of photoassimilates to CY-infected sieve tubes.

The question now arises, if the sugar upsurge is due to a higher activity of enzymes involved in oligosaccharide metabolism and/or to upregulation of transporters directing these substances to the SE/CC complexes in the midribs.

Increased levels of SE-restricted AtSUS5 and AtSUS6 expression (Fig. 7) suggest an enhanced need for sugar processing in SEs. Since CWINV1 and CWINV6 are not upregulated in infected mutant and wild-type plants (Fig. 7), apoplasmic breakdown of sucrose is likely not critical for the sugar supply of phytoplasmas. By contrast, sugar transport capacity to phytoplasmas (and thus to SEs) seems to be limited in wild-type plants. Without exception, transporters involved in sugar uptake by SE/CC complexes are upregulated in healthy mutant plants (Fig. 7), but not in wild-type plants. The higher expression rate, probably subject to effector control, renders it plausible that the better symplasmic mobility of phytoplasma effectors (Fig. 5) make them more effective in mutants.

AtSUC2 and AtSUC3, encode for transmembrane sucrose cotransporters localizing respectively in CCs and in SEs (Meyer et al., 2004). AtSUC2 and AtSUC3 are not only responsible for the sucrose loading, but are also involved in the sucrose retrieval along the transport phloem (Meyer et al., 2004; Gould et al., 2012).

AtSTP13 belongs to a family of genes encoding for hexose cotransporters. AtSTP13 basal resistance to necrotrophic fungi (i.e. Botrytis cinerea, Lemonnier et al., 2014) and extracellular bacteria (i. e. Pseudomonas syringae, Nørholm et al., 2006) by depriving these pathogens of modified sugar fluxes toward host cells. On the contrary, the overexpression of STP13 in plants infected with intracellular biotrophic pathogens provokes

413 increased host susceptibility (Huai et al., 2020), by promoting cytoplasmic hexose 414 accumulation. As phytoplasmas are biotrophic intracellular pathogens, it is conceivable 415 that the over-expression of AtSTP13 in CY-infected Atcals7ko plants could also indicate an 416 increased susceptibility of this line to phytoplasma attack, in comparison to the wild-type 417 (Bezrutczyk et al., 2018). Indeed, Atcals7ko line appears more affected by infection (i.e. 418 Fig. 2). 
As for AtSUCs, AtSWEET11 and AtSTP13 are also involved in the retrieval of sucrose along the transport phloem (Meyer et al., 2004; Gould et al., 2012). Taken together, the significant increase of AtSWEET11, AtSUC3 and AtSTP13 transcripts in the midribs of infected Atcals7ko plants indicates that sugar retrieval along the phloem path is highly activated in the host to fuel pathogen proliferation (Tadege et al., 1998). While AtSUC2, AtSUC3, and AtSTP3 are localized to the SE/CC complexes (Fig. 9), the cellular deployment of SWEET11 and SWEET12 is less certain. It must be underscored that midribs contain SE/CC complexes with a high degree of release and retrieval due to symplasmic isolation under source-limiting conditions. If SWEETs are responsible for sucrose supply of phytoplasma, they may mediate import at the SE/CC complex plasma membrane or export at the phloem parenchyma plasma membrane (Fig. 9).

AtSWEET11 and 12 were characterized as sucrose effluxers localized to the plasma membrane of phloem parenchyma cells with a role in feeding active transport into the SE/CC complex in the collection phloem (Chen et al., 2012). They are expressed in most Arabidopsis tissues (Braun, 2012), so, beyond phloem loading, they might play other roles (Breia et al., 2021). In the current understanding, deployment of AtSWEET11 and AtSWEET12 in phloem parenchyma of floral stems is quite meaningful (Le Hir et al., 2015). OsSWEETs are also tentatively localized to phloem parenchyma in the phloemunloading zone of rice (Milne et al., 2018), but StSWEET11 was proposed to be deployed in the plasma membrane of companion cells of potato transport phloem (Abelenda et al., 2019). With the present arguments in hand, it seems plausible that SWEET11 and SWEET12 are localized to the phloem parenchyma (Fig. 9).

The possibility that SWEETs determine plant susceptibility or resistance by the control of nutrient provision to pathogens has recently been discussed (Chen et al., 2010; Bezrutczyk et al. 2017; Breia et al., 2021). AtSWEET11 is a member of the clade III of the SWEET genes, involved in disease development (Li et al., 2018). Wipf et al., (2021) reported that AtSWEET11 interacts with AtRBOHD, a membrane NADPH oxidase producing reactive oxygen species, involved in defense-related processes. The constitutive downregulation of AtSWEET11 in the mutant line could be related to a less 448 effective capability to react to pathogen attacks. In this regard, the increased expression 449 level of AtSWEET11 in infected mutant plants (with the simultaneous over expression of 450 AtSUC3) could promote sucrose release towards the SE/CC complexes to support 


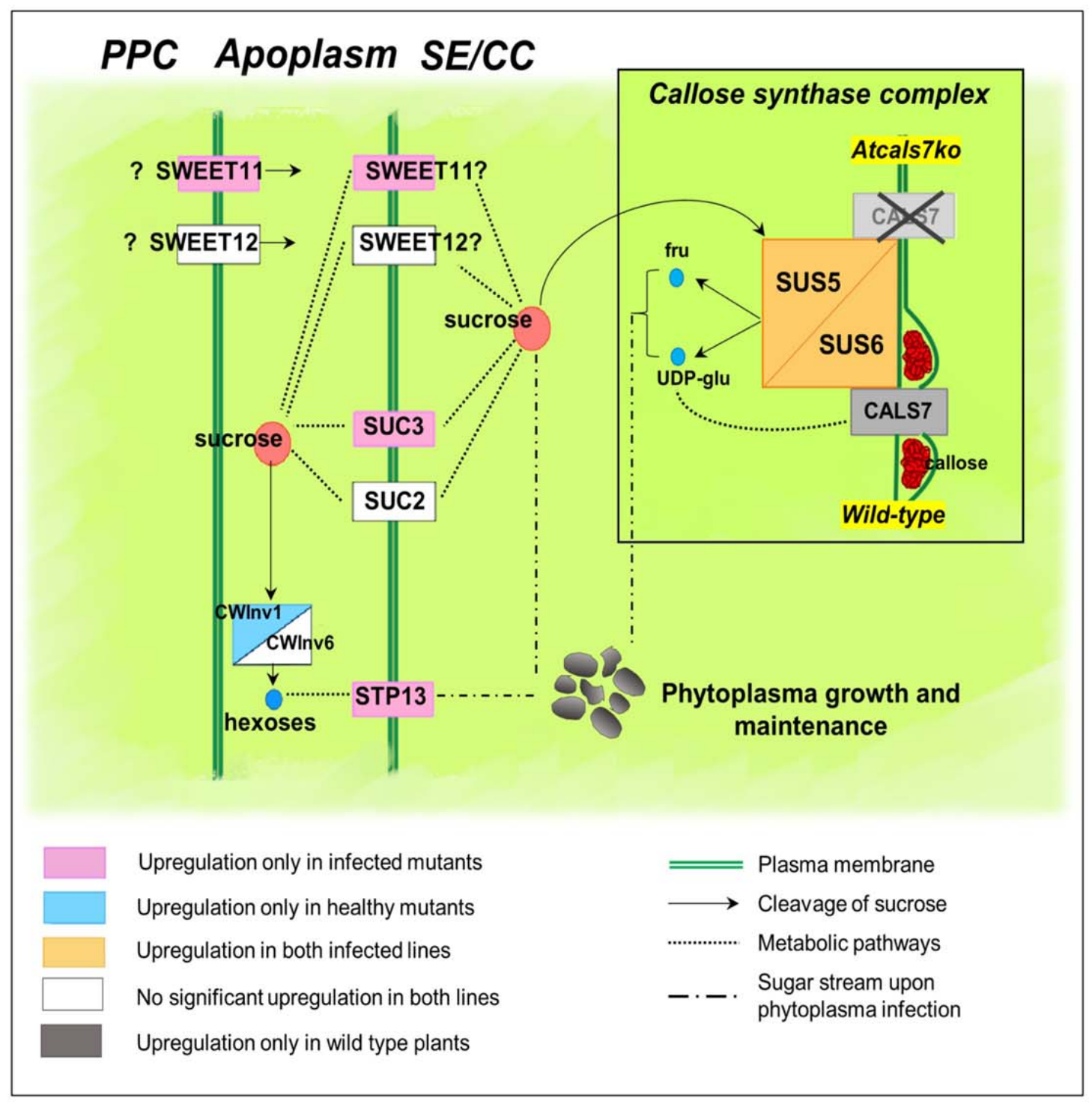

Fig. 9. Localization of the proteins involved in sugar metabolism and transport in the midribs of wild-type and Atcals7ko Arabidopsis and their expression in response to phytoplasma infection. In transport phloem, AtSUC2, AtSUC3, and AtSTP13 are localized to the plasma membrane of SE/CC complexes, while deployment of SWEET11 and SWEET12 is less certain, as they localized to the phloem parenchyma (Le Hir et al., 2015) but also to the companion cells (Abelenda et al., 2019). AtSUS5 and 6 are localized to the SE plasma membrane. Independently from the different protein localization, and regardless of the carbohydrate form (sucrose or hexoses), up-regulation of AtSUC3, AtSTP13 and AtSWEET11 in Atcals7ko plants would stimulate sucrose transport to SE/CC complexes (dotted lines), supporting phytoplasma maintenance (dashed lines) and giving rise to an increased susceptibility of the mutant line to the infection. 


\section{Concluding remarks}

454

455

456

457

458

459

460

461

462

463

464

465

466

467

468

469

470

471

472

473

474

475

476

477

478

479

480

481

482

483

AtCALS7 appears to have two layers of action as demonstrated by Atcals7ko mutants. As a first layer of action, knocking out AtCALS7 strongly restricts the supply of terminal sinks with resources and enhances the supply of axial sinks (Fig. 8). This results in a severely reduced growth of host plants. The second layer of action becomes manifest by an increased inability to counter infection due to an increased symplasmic connectivity in Atcals7ko mutants, which allows a better spread of phytoplasma effectors. The effectors probably stimulate the expression of genes involved in channeling resources towards the phytoplasmas residing in sieve tubes (Fig. 9). The enhanced withdrawal of sugar reserves from the host metabolism favours phytoplasma growth and an additional plant growth reduction in Atcals7ko mutants.

\section{Materials and methods}

\section{Plants and insect vectors}

Seeds of wild-type and Atcals7ko (SALK_048921) lines of Arabidopsis thaliana ecotype Col0 were provided by the Nottingham Arabidopsis Stock Centre (NASC). Sixtyfour plants were grown in a climate chamber for 40 days on 5:1 mixture of soil substrate and perlite and fertilized twice a month with an N-P-K liquid fertilizer, as described previously (Pagliari et al., 2017), 32 plants under short-day light conditions (9h $\mathrm{L} / 15 \mathrm{~h} \mathrm{D})$ at $18-20^{\circ} \mathrm{C}$, and 32 under long-day light conditions (14h L/10h D), at $23^{\circ} \mathrm{C}$ in order to develop the floral stem for the transport speed experiment.

Healthy colonies of Macrosteles quadripunctulatus were reared on Avena sativa in vented plexiglass cages in greenhouse (temperature of $20-22^{\circ} \mathrm{C}$ and short-day conditions $9 \mathrm{~h}$ L/15h D), according to Bosco et al. (1997). The last instar nymphs were transferred to Chrysanthemum carinatum plants infected with Chrysanthemum Yellows (CY) phytoplasma (Lee et al., 2003), a strain related to 'Candidatus Phytoplasma asteris' ('Ca. P. asteris', 16Srl-B subgroup), as the source of inoculum for a 7-day phytoplasma acquisition-access period (AAP). After a latent period (LP) of 18 days, insects were transferred to 40-day-old Arabidopsis plants for the 7-day inoculation-access period (IAP).

Eight wild-type and eight Atcals7ko plants were exposed to 3 infectious $M$. quadripunctulatus individuals (CY-infected plants). In addition, sixteen plants from both 
484 lines (8+8) were exposed to 3 healthy vectors as a control (healthy plants). Healthy

485 leafhoppers were collected from healthy colonies and were of the same age as the 486 infected ones. When symptoms on the infected plants grown under short-day conditions 487 were clearly visible, i.e. 26 days post inoculation (Pacifico et al., 2015; Pagliari et al., 488 2017), midribs from the leaves of the third rosette node (source leaves) were collected. For 489 phytoplasma detection and gene expression analyses, collected samples were 490 immediately frozen in liquid nitrogen and stored at $-80^{\circ} \mathrm{C}$ until use. For carbohydrate 491 analyses, midribs were immediately frozen in liquid nitrogen, freeze-dried and then stored 492 at $-80^{\circ} \mathrm{C}$ until use.

\section{Phloem transport speed measurement}

For phloem transport experiments, Arabidopsis plants were grown as reported above, under long-day light conditions $(14 \mathrm{~h} \mathrm{~L} / 10 \mathrm{~h} \mathrm{D})$, at $23^{\circ} \mathrm{C}$. Sixty-day-old healthy plants of both lines, were used for the measurement. Two x-ray photomultiplier tubes, $5.6 \mathrm{~cm}$ in diameter, (St. Gobain, Malvern, PA) were placed along the floral stem of both wild-type and Atcals7ko plants, leaving a 2-cm-long buffer zone between the detectors, while a third detector monitored the signal from the rosette.

Each detector was connected to an M4612 12-channel counter and counts of x-rays were logged with the manufacturer's software (Ludlum Measurements, Sweetwater, TX, USA). Plants were placed on a lead layer having a receptacle, under the same day length used for growing. After at least 8 hours of background measurement, $600 \mu \mathrm{l}$ of $\mathrm{NaH}^{14} \mathrm{CO}_{3}$ solution (specific activity: 40-60 $\mathrm{mCi}(1.48-2.22 \mathrm{GBq}) / \mathrm{mmol}$ ) were injected into the receptacle. Immediately thereafter, $1000 \mu \mathrm{l}$ of a saturated citric acid solution was also injected into the receptacle. Plants were tightly closed in a plastic bag, and were allowed to assimilate ${ }^{14} \mathrm{CO}_{2}$ for 2 hours. Then the bag was removed and the remaining gaseous radiolabelled isotope was flushed via the fume hood. The $\mathrm{x}$-ray detectors monitored the $\mathrm{x}-$ ray counts from the plant tissue every minute for 72 hours. The distance between the detectors was measured at the end of the experiment.

The data of the counts from each detector were handled with the <nmle> package in 512 Rstudio (Pinheiro et al., 2018). In accordance with previously published methods, the 513 Rstudio script was written to create a logistic function, and to fit the logistic function to the 514 data with time on the x-axis and the recorded counts on the y-axis (Vincent et al., 2019). 515 Xmid, which represents the time at the half-height of the logistic curve or the average time 
516 of arrival of ${ }^{14} \mathrm{C}$ in the phloem tissue near the $\mathrm{x}$-ray detector, was calculated for each

517 detector placed along the stem. The speed of translocation was calculated by dividing the

518 distance between the middle of the two detectors (in $\mathrm{cm}$ ) and the difference between the

519 Xmid timepoints from the detectors (in h). Both infected lines failed to normally develop

520 flowering stalks: stalk was very short in infected wild-type plants and absent in infected

521 Atcals7ko (Fig. 2C). For these reasons, it was not possible to calculate and compare sugar

522 translocation speed in the two infected Arabidopsis lines. For both wild-type and Atcals7ko

523 healthy plants 4 measurements were carried out, pairing 1 wild-type and 1 Atcals7ko

524 together in each labelling.

\section{Symptom description}

526 Symptom development was observed in healthy and CY-infected plants of both lines, 527 from the end of the inoculation period to the time of tissue harvest for different analyses, 528 i.e. when plants were ca. 70 days old. To evaluate the phenotypic differences between the 529 two lines and the differences due to phytoplasma infection, the rosette fresh weight was 530 measured in plants grown under short-day conditions using 10 healthy and $10 \mathrm{CY}$-infected 531 plants per line. The day before sampling, soil was saturated with water. Rosettes were 532 then cut at the plant collar level and the fresh weight of each biological replicate was 533 measured. Moreover, the length of the floral stalk was measured in plants grown under 534 long-day light conditions (see above), using 8 healthy and 8 CY-infected plants per line. 535 Statistical analyses were performed using RStudio software Version 1.1.456 (RStudio 536 Team 2020, Boston, MA). The normal distribution was checked with the Shapiro-Wilk test. 537 Significant differences among the group means were determined with a two-way ANOVA 538 and post-hoc comparisons between all groups were made with Tukey's test with $\mathrm{P}<0.05$.

539 Phytoplasma detection and quantification

540 To check the phytoplasma titre in wild-type and Atcals7ko CY-infected plants, 541 genomic DNA was extracted from $200 \mathrm{mg}$ of fresh leaf midrib tissue, according to Doyle 542 and Doyle protocol, (1990) modified by Martini et al., (2009). The ribosomal protein gene $543 \mathrm{rp} / \mathrm{V}(\mathrm{rp} / 22)$ was chosen as a target for the amplification of CY phytoplasma DNA using the 544 primer pair rp(I-B)F2/rp(I-B)R2 (Lee et al., 2003; Pagliari et al., 2017) and a CFX96 realtime PCR detection system (Bio-Rad Laboratories, Richmond, CA, USA). A standard

546 curve was established by 10-fold serial dilutions of a plasmid DNA containing the $1260 \mathrm{bp}$ 547 ribosomal protein fragment from CY phytoplasma, amplified with the primer pair $548 \mathrm{rpF} 1 \mathrm{C} / \mathrm{rp}(\mathrm{I}) \mathrm{R} 1 \mathrm{~A}$ (Martini et al., 2007). Real-time PCR mixture and cycling conditions were 
previously described (Pagliari et al., 2017). The phytoplasma titre was expressed as the number of CY-phytoplasma genome units (GUs) per mg of fresh leaf sample to normalize the data. Statistical significance of the quantitative differences between phytoplasma populations was calculated by analysis of three replicates of 8 plants per line. Statistical analyses were performed using RStudio software version 1.1.456 (2009-2018 RStudio, Inc. Boston, MA). The normal distribution was checked with Shapiro-Wilk test. Significant differences among the means were determined using the Kruskal-Wallis non-parametric test with $\mathrm{P}<0.05$.

\section{Gene expression analyses}

Total RNA was extracted from $100 \mathrm{mg}$ of leaf midrib powder, from 5 plants for the four experimental conditions, obtained by grinding in liquid nitrogen and using a SpectrumTM Plant Total RNA kit (Sigma-Aldrich, Merck KGaA, Darmstadt, DE) according to the manufacturer's instructions. The RNA reverse-transcription was carried out using a QuantiTectReverse Transcription Kit (Qiagen N.V., Hilden, DE) following the manufacturer's instructions. The expression of the genes, reported in Table 1, was analysed in healthy and CY-infected midribs by real-time experiments, performed on a CFX96 real-time PCR detection system (Bio-Rad Laboratories). The reference gene was selected by comparing the AtUBC9 (ubiquitin conjugating enzyme 9), AtTIP41 (TIP41-like family protein), AtSAND (SAND family protein), and AtUBQ10 (polyubiquitin 10) gene expression. The gene stability values (M values) were calculated according to the geNorm program (Pagliari et al., 2017). AtUBC9 gene was the most stably expressed gene and so the most suitable as a reference gene $(M=0.44)$. SsoFast EvaGreen Supermix 2x (Bio-Rad Laboratories Inc., Hercules, CA, USA) and cDNA obtained from 5 ng of RNA and specific primers (Table1) were used in a total volume of $10 \mu \mathrm{l}$. Under these conditions, the primer pair efficiency was evaluated as described by Pfaffl (Pfaffl, 2001) using standard curves of different dilutions of pooled CDNA. PCR was performed as described in Pagliari et al., (2017), with three technical repeats. A mean normalized expression (MNE) for each gene of interest (Muller et al., 2002) was calculated by normalizing its mean expression level to the level of the UBC9 gene. Five individuals were used for the gene MNE determination. Statistical analyses were performed using RStudio software Version 1.1.456 (2009-2018 RStudio, Inc. Boston, MA). The normal distribution was checked with Shapiro-Wilk test. Significant differences among the means were determined by a two-way ANOVA and posthoc comparisons between all groups were made with Tukey's test with $\mathrm{P}<0.05$. 
582

583

584

585

586

587

588

589

590

591

592

593

594

595

596

597

598

599

600

601

602

603

604

605

606

607

608

609

610

611

612

613

614

\section{Transmission electron microscopy}

To observe phloem ultrastructure in the midrib, samples were prepared for microscopic analyses as reported by Pagliari et al., (2016). For each condition, at least five segments (10 $\mathrm{mm}$ in length) of the mature leaf midrib from 5 individuals were submerged in MES buffer (10mM NaOH-2-( $N$-morpholino) ethanesulfonic acid, $2 \mathrm{mM} \mathrm{CaCl}_{2}, 1 \mathrm{mM}$ $\mathrm{MgCl}_{2}, 0.5 \mathrm{mM} \mathrm{KCl}$ and $200 \mathrm{mM}$ mannitol, $\mathrm{pH} 5.7$ ) for $2 \mathrm{~h}$ at room temperature. Samples were fixed in a solution of 3\% paraformaldehyde and $4 \%$ glutaraldehyde for $6 \mathrm{~h}$, the solution was refreshed every 30 minutes. After rinsing, samples were post-fixed overnight with $2 \%(\mathrm{w} / \mathrm{v}) \mathrm{OsO}_{4}$, dehydrated with an ethanol gradient and transferred to pure propylene oxide. Midrib segments were then embedded in Epon/Araldite epoxy resin (Electron Microscopy Sciences, Fort Washington, PA, USA). Ultra-thin sections were collected on uncoated copper grids, stained with UAR-EMS (uranyl acetate replacement stain, Electron Microscopy Sciences) and then observed under a PHILIPS CM 10 (FEI, Eindhoven, The Netherlands) transmission electron microscope (TEM), operated at $80 \mathrm{kV}$, and equipped with a Megaview G3 CCD camera (EMSIS GmbH, Münster, Germany). Five non-serial cross-sections from each sample were analysed.

\section{Confocal laser scanning microscopy}

In both plant groups, healthy or CY-infected, callose deposits, associated with plasmodesmata or sieve plates, were identified in leaf epidermis, midrib cortical parenchyma, and SE/CC areas, using aniline blue staining and Confocal Laser Scanning Microscopy (CLSM). Callose was quantified in situ by measuring the aniline blue fluorescence intensity (Levy et al., 2007). For analysis of the epidermis, whole leaves from the third rosette node were collected in $95 \% \mathrm{EtOH}$ solution and stored for at least 2 hours at room temperature, as described by Zavaliev and Epel (2015). Samples were rehydrated with distilled water with $0.01 \%$ Tween-20 for 1 hour, then placed in a tube with aniline blue solution $\left(0.01 \mathrm{M} \mathrm{K}_{3} \mathrm{PO}_{4}, \mathrm{pH}=12\right)$. Tubes with samples were placed in a vacuum desiccator for 10 minutes, then incubated under aluminum foil for 2 hours. Two leaves from three plants for every condition (i.e. wild-type or Atcals7ko, healthy or CY-infected) were observed. For every leaf at least 20 images were taken on a single-plane, using a Leica SP8 LSCM (Leica Microsystems Inc., Buffalo Grove, IL, USA) equipped with 40x oil immersion objective. Aniline blue fluorescence was excited with $405 \mathrm{~nm}$ diode laser and emission was detected at 475-525nm. Optimal conditions for microcopy were previously determined using healthy wild-type samples. 
615 To analyze callose deposits in the midribs, both in the SE/CC area and in the surrounding

616 midrib cortical parenchyma, leaf midribs were collected in a 95\% EtOH solution and stored

617 at least $2 \mathrm{~h}$ at room temperature. Hand-made cross sections, cut with a razor blade, were

618 incubated for 5 minutes in aniline blue solution. The sections were rinsed with $0.01 \%$

619 Tween-20 solution. For each experimental condition at least 10 non-serial sections from 5

620 different specimens were observed using a Leica SP8 CLSM with a 10x objective. Aniline

621 blue fluorescence was excited with a $405 \mathrm{~nm}$ diode laser and emission was detected at

622 475-525 nm. Optimal conditions for microscopy were previously determined by using

623 healthy wild-type samples.

624 Image analysis was performed using FIJI software (Schindelin et al., 2012). For the 625 analyses of epidermis and midrib cortical parenchyma, images were treated following the 626 protocol by Zavaliev and Epel, (2015). A macro separated the fluorescent signal in the 627 region of interest from the background using an auto local threshold with the Phansalkar 628 algorithm with radius $=1$ for epidermal tissue, and the Bernsen algorithm with radius $=10$ 629 for midrib cortical parenchyma. For cortical parenchyma, an area of interest of $45000 \mu \mathrm{m}^{2}$ 630 was analyzed.

631 Fluorescent dots (indicating callose deposits) in the SE/CC area were analyzed as 632 previously reported by Pagliari et al., (2016). For every group of samples, the mean gray 633 value (callose intensity), the number of callose deposits for each square millimeter and the 634 integrated density (sum of intensity within the area of the region of interest) were 635 considered. Data obtained by analyses were handled using RStudio software (2009-2018 636 RStudio, Inc. Boston, MA). Normality of the data was checked with the Shapiro-Wilk test, 637 outliers were removed according to the RStudio function (boxplot(data \$variable, 638 plot=FALSE)\$out), and data were normalized, where necessary, with Box-Cox 639 transformation. A two-way analysis was performed followed by a post-hoc comparison of 640 all pairs with Tukey's test, with $\mathrm{P}<0.05$.

\section{Sugar quantification}

642 Authentic standards of sugars (rhamnose, arabinose, fructose, glucose, maltose, 643 sucrose, and melibiose) and sugar alcohols (glycerol, myoinositol, arabitol, and sorbitol) 644 were purchased from Sigma-Aldrich (St. Louis, MO, USA). Internal standards including 645 fructose $-{ }^{13} \mathrm{C} 6$ (for sugars) and sorbitol- ${ }^{13} \mathrm{C} 6$ (for sugar alcohols) were obtained from 646 Toronto Research Chemicals (Toronto, ON, Canada) and Sigma-Aldrich (St. Louis, MO, 
647 USA), respectively. Water, acetonitrile, methanol, and formic acid were of LC-MS grade, 648 and were purchased from Fisher Scientific (Fair Lawn, NJ, USA). Stock solutions of each analyte and internal standard were prepared at a concentration of $10,000 \mu \mathrm{g} / \mathrm{ml}$ in water, or methanol. Working standard solutions were prepared by diluting and mixing each stock solutions with $90 \%$ methanol (water/methanol $=10 / 90$, v/v). The stock and working solutions were stored at $-80^{\circ} \mathrm{C}$. Freeze-dried leaf midribs were stored at $-80^{\circ} \mathrm{C}$ until use. Ten milligram of ground samples treated with $0.05 \mathrm{ml}$ of internal standard solution (50 $\mu \mathrm{g} / \mathrm{ml}$ sorbitol-13C6 and $200 \mu \mathrm{g} / \mathrm{ml}$ fructose-13C6 in $90 \%$ acetonitrile (water/acetonitrile $=$ $10 / 90, \mathrm{v} / \mathrm{v}$ ) was extracted with $0.95 \mathrm{ml}$ of $90 \%$ acetonitrile (water/acetonitrile $=10 / 90, \mathrm{v} / \mathrm{v}$ ) (total volume: $1 \mathrm{ml}$ ) by ultra-sonication for $30 \mathrm{~min}$, followed by agitation for $30 \mathrm{~min}$. After centrifugation $\left(20,000 \mathrm{~g}, 5 \mathrm{~min}, 4^{\circ} \mathrm{C}\right)$, supernatant was further filtered through $0.22 \mu \mathrm{m}$ nylon filter, and was injected into LC-MS/MS for analysis. The extraction was performed in triplicate using 4 biological replicates. Data obtained by analyses, were handled using RStudio software. Normality of the data was checked with Shapiro-Wilk test, outliers were removed, and data were normalized, where necessary, with Box-Cox transformation. A two-way analysis was performed followed by post-hoc pairwise comparison of all groups with Tukey's test, with $\mathrm{P}<0.05$.

\section{Acknowledgements}

This work was funded by the University of Udine, through the Department of Agriculture, 668 Food, Environment and Animal Sciences (Di4A), Project Start-up 2018. The authors thank Professor Domenico Bosco (University of Torino, Italy) for making available the CY phytoplasma strain and for his advice on insect rearing.

673 Fig. 1. Phloem transport speed in wild-type and Atcals7ko Arabidopsis lines. Carbohydrate 674 translocation speed along the phloem, measured with ${ }^{14} \mathrm{C}$ isotope. The speed is expressed as $\mathrm{cm} / \mathrm{h}$ and it is 675 calculated by average time of arrival of the ${ }^{14} \mathrm{C}$ label in the stem phloem tissue near the $\mathrm{x}$-ray detector. 676 Statistical analysis was performed using the Tukey HSD test as the post hoc test in an one-way ANOVA. 677 Different letters $(a, b)$ above the bars indicate significant differences, with $P<0.05$. Error bars indicate the 678 Standard Error of the Mean of 4 biological replicates for each condition. 
679 Fig. 2. Plant phenotype and phytoplasma titre in wild-type and Atcals7ko line. Representative images

680

681

682

683

684

685

686

687

688

689

690

691

692

693

694

695

696

697

698

699

700

701

702

703

704

705

706

707

708

709

710

711

712 of healthy and CY-infected wild-type and Atcals7ko plants. Following CY-infection, at 20 days after the inoculation access period (IAP), both plant groups showed yellowish small leaves. Leaves emerged after phytoplasma inoculation were shorter, with thicker main vein and smaller petiole (A). Regardless phytoplasma infection, rosette fresh weight is significantly reduced in Atcals7ko plants in comparison with wild-type (B). In 60-day-old Arabidopsis plants, grown under long day conditions, stem was well developed in healthy wild-type but reduced in length in Atcals7ko plants (C, D). Following CY infection, stem length resulted strongly reduced in wild-type individuals and absent in the Atcals7ko mutants (C, D). Although not significantly different, phytoplasma titre increases in the mutant line in comparison to the wild type (E). Different letters indicate different means according to the non-parametric Kruskal-Wallis post hoc test, $\mathrm{P}<0.05$. Error bars indicate Standard Error of the Mean of 8 biological replicates for each condition. Plant weight (B) 20 days IAP expressed as mg of fresh tissue. Statistical analysis was performed using the Tukey HSD test as the post hoc test in a two-way ANOVA. Different letters $(a, b)$ above the bars indicate significant differences, with $P<0.05$. Error bars indicate the Standard Error of the Mean of 8 biological replicates for each condition.

Fig. 3. Representative TEM micrographs of the sieve elements of healthy and CY-infected Arabidopsis lines. A-H. Cross-sections of midribs from healthy (A-D) and infected (E-H) wild-type Arabidopsis leaves. Healthy samples present unaltered sieve elements and companion cells (A), with a regular shape and no signs of necrosis or subcellular aberrations. The sieve pores, at both lateral (B) and ordinary (C) sieve plates, are not constricted by callose collars, pore-plasmodesma units are open and show their typical branched shape with sieve endoplasmic reticulum located in the proximity of the orifice (D). In infected midribs, numerous phytoplasmas are visible inside the sieve elements $(E)$ and at the sieve plate $(\mathbf{F})$. Lateral sieve plates have sieve pores mostly open (F), whereas in ordinary sieve plates (G) pores are constricted by callose depositions. Pore-plasmodesma units display a similar morphology as in healthy samples, with a thin callose line at the sieve-element side (H). I-P. Cross-sections of midribs from healthy (IL) and CY-infected (M-P) Atcals7ko Arabidopsis leaves. In healthy Atcals7ko samples, phloem cells are apparently well structured (I), but the sieve plates show aberrant morphology $(\mathbf{J}, \mathbf{K})$. Pores lack callose and appear not fully developed $(\mathbf{J}, \mathbf{K})$. Pore-plasmodesma units are branched, similar as in wild-type samples (L). In CY-infected Atcals7ko samples, many phloem cells evidence thick cell walls (M), others are collapsed $(\mathbf{N})$. Sieve plates are deformed, thickened $(\mathbf{N}, \mathbf{O})$ and pores are filled by electron-opaque material $(\mathbf{N})$. Poreplasmodesma units appeared large, without well-defined branches $(\mathbf{P})$.

Arrows: callose; CC: companion cell; PPC: phloem parenchyma cell; pl: plastid; PPU: pore-plasmodesma unit; SE: sieve element; ser: sieve element reticulum; SP: sieve plate; star: phytoplasmas. Bars correspond to $1 \mu \mathrm{m}$.

Fig. 4. Single-plane confocal micrographs and imaging of callose deposits at plasmodesmata in phloem area and in parenchyma of healthy and CY-infected Arabidopsis midribs. Fluorescent spots indicate callose depositions in the phloem area of healthy $(\mathbf{A})$ and $\mathrm{CY}$-infected $(\mathbf{B})$ wild-type samples. The fluorescence intensity $(E)$, the number $(\mathbf{F})$ and the integrated density [i.e. the sum of the intensity in the region of interest $(\mathrm{ROI})]$ of the deposits $(\mathbf{G})$ is significantly higher in the infected midribs compared to the 
718

719

720

721

722

723

724

725

726

727

728

729

730

731

732

733

734

735

736

healthy ones. No signal is visible in Atcals7ko midribs (C, D). Punctate dots, indicating callose deposits at plasmodesmata are visible in the midrib parenchyma of all samples (A-D). In the ROI, fluorescence intensity $(\mathbf{H})$ and number of dots $(\mathbf{I})$ are not different in healthy samples, but the integrated density (i.e. the sum of the intensity within the area of the region of interest, $\mathrm{ROI}$ ) is significantly higher in the mutant line than in the wild-type (J). Following infection, the fluorescence intensity and the integrated density of the signal in the $\mathrm{ROI}$ significantly increased only in wild-type samples. In the pictures, stars indicate phloem and triangle indicate xylem. Statistical analysis was performed using the Tukey HSD test as the post hoc test in a twoway ANOVA. Different letters ( $a, b, c, d)$ above the bars indicate significant differences, with $P<0.05$. Boxplots were obtained from three biological replicates for each condition and almost 10 technical replicates for each sample. Bars correspond to $100 \mu \mathrm{m}$.

Fig. 5. Single-plane confocal micrographs and imaging of callose deposits at plasmodesmata in epidermal cells. Aniline blue fluorescent dots (A-D), their intensity $(\mathbf{E})$, number $(\mathbf{F})$ and integrated density (i.e. the sum of the intensity within the area of the region of interest, ROI, $\mathbf{G}$ ) were assayed at leaf epidermis level in healthy and infected samples of both lines. In healthy samples, the intensity $(\mathbf{E})$ and the number of fluorescent dots (F), and the integrated density (G) are lower in Atcals7ko line compared to the wild-type. Following CY-infection the three parameters significantly increased only in Atcals7ko line. Statistical analysis was performed using the Tukey HSD test as the post hoc test in a two-way ANOVA. Different letters $(a, b, c)$ above the bars indicate significant differences, with $P<0.05$. Boxplots were obtained from three biological replicates for each condition and almost 10 technical replicates for each sample. Bar corresponds to $50 \mu \mathrm{m}$.

Fig. 6. Sugar quantification in the midribs of healthy and infected Arabidopsis lines. Sucrose (A), glucose (B), fructose (C), myoinositol (D), sorbitol (E), raffinose $(\mathbf{F})$ and arabinose (G) were quantified in midribs of the two different lines, healthy or CY-infected. Data obtained were expressed as analyte/IS peak area ratio. Statistical analysis was performed using the Tukey HSD test as the post hoc test in a two-way ANOVA. Different letters $(a, b)$ above the bars indicate significant differences, with $P<0.05$. Error bars indicate the Standard Error of the Mean of 4 biological replicates for each condition run in triplicate.

Fig. 7. Transcript profiling of genes involved in phloem callose synthesis and sugar transport and metabolism. A. Expression level of phloem callose synthase gene (AtCALS7) in healthy and CY-infected wild-type plants. B-F. Transcript profiling of sucrose synthases AtSUS5 and AtSUS6 (B), sucrose transporters AtSUC2 and AtSUC3 (C), SWEET sugar facilitators AtSWEET11 and AtSWEET12 (D), cell wall invertases AtCWINV1 and AtCWINV6 (E), the hexose transporter AtSTP13 (F). Healthy and infected plants belonging to the two lines were compared. Expression values were normalized to the UBC9 transcript level, arbitrarily fixed at 100, then expressed as mean normalized expression $\pm S D$ (transcript abundance). Statistical analysis was performed using the Tukey HSD test as the post hoc test in a two-way ANOVA. Different letters $(a, b, c, d)$ above the bars indicate significant differences, with $P<0.05$. Error bars indicate the Standard Error of the Mean of 5 biological replicates for each condition run in triplicate.

Fig. 8. Hypothetical model for the impact of functional (wild-type line) or aberrant (Atcals7ko line) sieve plates on the photoassimilate investment into terminal or axial sinks. The red arrowheads quantify the extent of investment in terminal sinks, which is correlated to the translocation speed. The size of 
756 the horizontal black arrows quantifies photoassimilates which are invested in axial sinks. Photoassimilate

757 investment into terminal sinks is lower in the Atcals7ko line, due to aberrant sieve plates, which results in

758 more escape of carbohydrates along the pathway towards the axial sinks. In case of phytoplasma infection,

759 photoassimilate investment into terminal sinks could be more affected, favoring not only the axial sink

760 proliferation, but also the phytoplasma (additional sink) nourishment.

761 Fig. 9. Localization of the proteins involved in sugar metabolism and transport in the midribs of wild-

762 type and Atcals7ko Arabidopsis and their expression in response to phytoplasma infection. In

763 transport phloem, AtSUC2, AtSUC3, and AtSTP13 are localized to the plasma membrane of SE/CC

764 complexes, while deployment of SWEET11 and SWEET12 is less certain, as they localized to the phloem

765 parenchyma (Le Hir et al., 2015) but also to the companion cells (Abelenda et al., 2019). AtSUS5 and 6 are

766 localized to the SE plasma membrane. Independently from the different protein localization, and regardless

767 of the carbohydrate form (sucrose or hexoses), up-regulation of AtSUC3, AtSTP13 and AtSWEET11 in

768 Atcals7ko plants would stimulate sucrose transport to SE/CC complexes (dotted lines), supporting

769 phytoplasma maintenance (dashed lines) and giving rise to an increased susceptibility of the mutant line to

770 phytoplasma infection. 
Table 1: List of the genes analyzed in this work. For every gene the locus, the primer sequences, the accession number (NCBI)and

772 the function has been reported.

\begin{tabular}{|c|c|c|c|c|c|}
\hline Gene & Locus & $\begin{array}{l}\text { Forward } \\
\text { (sequence } 5^{\prime}-3^{\prime} \text { ) }\end{array}$ & $\begin{array}{c}\text { Reverse } \\
\text { (sequence } 5^{\prime}-3^{\prime} \text { ) }\end{array}$ & $\begin{array}{l}\text { Accession } \\
\text { NCBI }\end{array}$ & Functions \\
\hline AtCal7 & At1g06490 & TTATTTGTCGTCTGCGGCCT & TCCTTTGAACACGCTACGCA & NM_100528.2 & $\begin{array}{l}\text { Callose deposition at the sieve pores level (Barratt et } \\
\text { al., 2011; Xie et al., 2011) }\end{array}$ \\
\hline AtSUS5 & At5g37180 & TGGAAGCAAAGAGAGGGCTG & CCAGGAGCTGCGATGTTGAA & NM_123077.2 & \multirow{2}{*}{$\begin{array}{l}\text { Sucrose synthases responsible for the cleavage of the } \\
\text { sucrose to provide UDP-glucose to several pathways } \\
\text { in a reversible reaction (i.e. callose synthesis and } \\
\text { cellulose synthesis). Peculiar of the sieve element. } \\
\text { (Barratt et al., 2009) }\end{array}$} \\
\hline AtSUS6 & At1g73370 & ACATAGCCAAGGAGCTTCGC & CTTGAGCCGAGTTAGCACCA & NM_001198461.1 & \\
\hline AtCWINV1 & At3g13790 & ACTCGGCTAAGAACCGGAGA & CGGACCAGCCTTTCTCAACA & NM_001338080.1 & \multirow[b]{2}{*}{$\begin{array}{l}\text { They irreversibly catalyze the cleavage of the } \\
\text { extracellular sucrose into glucose and fructose } \\
\text { (Roitsch and González, 2004; Hirose et al., 2008), and } \\
\text { are typically expressed in tissues where phloem } \\
\text { unloading follows an apoplasmic pathway (Ruan, } \\
\text { 2014). Localization: cell wall. CWINV1 is expressed in } \\
\text { almost all the tissues, CWINV6 is more expressed in } \\
\text { the leaves (Sherson et al., 2003). They could have a } \\
\text { role in defense against pathogens (Fotopoulos et al., } \\
2003 \text { ). }\end{array}$} \\
\hline AtCWINV6 & At5g11920 & GTCACAGTTGTTGCCGAACC & GGCGGAACCATCACAGGATT & NM_001343222.1 & \\
\hline AtSUC2 & At1g22710 & GTCGCTGGAGCTGGTTTAGT & TATCGCTATGGCTCGCGTTT & NM_102118.4 & \multirow{2}{*}{$\begin{array}{l}\text { Sucrose and } \mathrm{H}^{+} \text {symporters, working against } \\
\text { concentration. AtSUC2 seems to be the most } \\
\text { expressed in Arabidopsis. Localization: companion } \\
\text { cells. (Durand et al., 2018) }\end{array}$} \\
\hline AtSUC3 & At2g02860 & TGTTCTGCTTGTGGATGGCT & ACATGCAGCACAACATGCTC & NM_201675.2 & \\
\hline AtSWEET11 & At3g48740 & AGGCACAGTTTCATCCCCTG & TGCTTGCCATGTTTAGGGGT & NM_114733.4 & \multirow[b]{2}{*}{$\begin{array}{l}\text { Uniporters playing an important role in the efflux of } \\
\text { sucrose, glucose and fructose (Le Hir et al., 2015) from } \\
\text { PPCs towards the apoplasmic space (Zhang, 2018), } \\
\text { and are responsible for the step before sucrose } \\
\text { phloem-loading mediated by AtSUC2/H+ symporter } \\
\text { (Braun et al., 2012; Chen, 2014). AtSWEET11 and } \\
\text { AtSWEET } 12 \text { are localized in the phloem parenchyma } \\
\text { with a probable regulation by Ca2+ ions. (Zhang, } \\
\text { 2018). }\end{array}$} \\
\hline AtSWEET12 & At5g23660 & CCCGGAACCAAAGATCGACA & GCACGGGAGAGAGGAAAACA & NM_122271.3 & \\
\hline AtSTP13 & At5g26340 & ATAGGTGTGGCTCTCAACGC & GACAAGAACAACGGAACGGC & NM_122535.4 & $\begin{array}{l}\text { High affinity hexose transporter, probably related to } \\
\text { vascular tissue, is confers resistance in case of rust. Its } \\
\text { activity increase in in presence of flagellin. (Julius et }\end{array}$ \\
\hline
\end{tabular}


al., 2017)

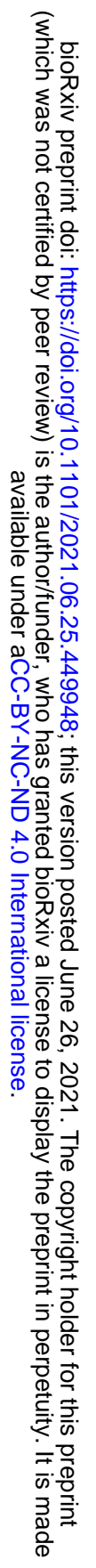


bioRxiv preprint doi: https://doi.org/10.1101/2021.06.25.449948; this version posted June 26, 2021. The copyright holder for this preprint (which was not certified by peer review) is the author/funder, who has granted bioRxiv a license to display the preprint in perpetuity. It is made available under aCC-BY-NC-ND 4.0 International license.

774

775 


\section{Parsed Citations}

Abelenda, J.A, Bergonzi, S., Oortwijn, M., Sonnewald, S., Du, M., Visser, R.G., Sonnewald, U., Bachem, C.W. (2019) Source-sink regulation is mediated by interaction of an FT homolog with a SWEET protein in potato. Current Biology 29, $1178-1186$.

Google Scholar: Author Only Title Only Author and Title

Abou-Saleh, R. H., Hernandez-Gomez, M. C., Amsbury, S., Paniagua, C., Bourdon, M., Miyashima, S., Helariutta, Y., Fuller, M., Budtova, T., Connell, S.D., Ries, M.E., Benitez-Alfonso, Y. (2018) Interactions between callose and cellulose revealed through the analysis of biopolymer mixtures. Nature communications, 9(1), 1-13.

Google Scholar: Author Only Title Only Author and Title

Albertazzi, G., Milc, J., Caffagni, A, Francia, E., Roncaglia, E., Ferrari, F., Tagliafico, E., Stefani, E., Pecchioni, N. (2009) Gene expression in grapevine cultivars in response to Bois Noir phytoplasma infection. Plant Science 176, $792-804$.

https://doi.org/10.1016/j.plantsci.2009.03.001

Google Scholar: Author Only Title Only Author and Title

Alma, A, Lessio, F., Nickel, H. (2019) Insects as phytoplasma vectors: ecological and epidemiological aspects, in: Phytoplasmas: Plant Pathogenic Bacteria-II. Springer, pp. 1-25.

Google Scholar: Author Only Title Only Author and Title

Barratt, D.H.P., Derbyshire, P., Findlay, K., Pike, M., Wellner, N., Lunn, J., Feil, R., Simpson, C., Maule, AJ., Smith, AM. (2009) Normal growth of Arabidopsis requires cytosolic invertase but not sucrose synthase. Proceedings of the National Academy of Sciences 106, 13124-13129. https://doi.org/10.1073/pnas.0900689106

Google Scholar: Author Only Title Only Author and Title

Barratt, D.H.P., Kölling, K., Graf, A, Pike, M., Calder, G., Findlay, K., Zeeman, S.C., Smith, AM. (2011) Callose Synthase GSL7 Is Necessary for Normal Phloem Transport and Inflorescence Growth in Arabidopsis. Plant Physiol. 155, 328-341.

https://doi.org/10.1104/pp.110.166330

Google Scholar: Author Only Title Only Author and Title

Bernardini, C., Pagliari, L., De Rosa, V., Ameida-Trapp, M., Santi, S., Martini, M., Buoso, S., Loschi, A, Loi, N., Chiesa, F., Mithöfer, A, van Bel, AJ.E., Musetti, R. (2020) Pre-symptomatic modified phytohormone profile is associated with lower phytoplasma titres in an Arabidopsis seor1ko line. Sci Rep 10, 14770. https://doi.org/10.1038/s41598-020-71660-0

Google Scholar: Author Only Title Only Author and Title

Bethke, G., Thao, A, Xiong, G., Li, B., Soltis, N.E., Hatsugai, N., Hillmer, R.A, Katagiri, F., Kliebenstein, D.J., Pauly, M. (2016) Pectin biosynthesis is critical for cell wall integrity and immunity in Arabidopsis thaliana. The Plant Cell 28, 537-556.

Google Scholar: Author Only Title Only Author and Title

Bezrutczyk, M., Hartwig, T., Horshman, M., Char, S.N., Yang, J., Yang, B., Frommer, W.B., Sosso, D. (2017) Impaired phloem loading in genome-edited triple knock-out mutants of SWEET13 sucrose transporters. bioRxiv 197921.

Google Scholar: Author Only Title Only Author and Title

Bezrutczyk, M., Yang, J., Eom, J., Prior, M., Sosso, D., Hartwig, T., Szurek, B., Oliva, R., Vera-Cruz, C., White, F.F. (2018) Sugar flux and signaling in plant-microbe interactions. The Plant Journal 93, 675-685.

Google Scholar: Author Only Title Only Author and Title

Bieniawska, Z, Paul Barratt, D.H., Garlick, AP., Thole, V., Kruger, N.J., Martin, C., Zrenner, R., Smith, AM. (2007) Analysis of the sucrose synthase gene family in Arabidopsis: The sucrose synthase gene family in Arabidopsis. The Plant Journal 49, $810-828$.

https://doi.org/10.1111/j.1365-313X.2006.03011.x

Google Scholar: Author Only Title Only Author and Title

Bolouri Moghaddam, M.R., Van den Ende, W. (2012) Sugars and plant innate immunity. Journal of experimental botany 63, $3989-3998$.

Google Scholar: Author Only Title Only Author and Title

Bolouri-Moghaddam, M.R., Le Roy, K., Xiang, L., Rolland, F., Van den Ende, W. (2010) Sugar signalling and antioxidant network connections in plant cells. The FEBS journal 277, 2022-2037.

Google Scholar: Author Only Title Only Author and Title

Bolton, M.D. (2009) Primary metabolism and plant defense-fuel for the fire. Molecular plant-microbe Interactions $22,487-497$.

Google Scholar: Author Only Title Only Author and Title

Bonke, M., Thitamadee, S., Mähönen, AP., Hauser, M.-T., Helariutta, Y. (2003) APL regulates vascular tissue identity in Arabidopsis. Nature 426, 181-186.

Google Scholar: Author Only Title Only Author and Title

Bosco, D., Minucci, C., Boccardo, G., Conti, M. (1997) Differential acquisition of chrysanthemum yellows phytoplasma by three leafhopper species. Entomologia Experimentalis et Applicata 83, 219-224. https://doi.org/10.1046/j.1570-7458.1997.00175.x

Google Scholar: Author Only Title Only Author and Title

Braun, D.M. (2012) SWEET! The pathway is complete. Science 335, 173-174.

Google Scholar: Author Only Title Only Author and Title 

available under aCC-BY-NC-ND 4.0 International license.

Braun, E. J., \& Sinclair, W. A (1978) Translocation in phloem necrosis-diseased American elm seedlings. Phytopathology, 68, $1733-1737$. Google Scholar: Author Only Title Only Author and Title

Breia, R., Conde, A, Badim, H., Fortes, AM., Gerós, H., Granell, A (2021) Plant SWEETs: from sugar transport to plant-pathogen interaction and more unexpected physiological roles. Plant Physiology.

Google Scholar: Author Only Title Only Author and Title

Cao, X.B., Fan, G.Q., Dong, Y.P., Zhao, ZL., Deng, M.J., Wang, Z, Liu, W. S. (2017) Proteome profiling of Paulownia seedlings infected with phytoplasma. Frontiers in Plant Science. 8, 342

Google Scholar: Author Only Title Only Author and Title

Cao, Y., Fan, G., Wang, Z, Gu, Z (2019) Phytoplasma-induced changes in the acetylome and Succinylome of Paulownia tomentosa provide evidence for involvement of acetylated proteins in Witches' Broom Disease. Mol Cell Proteomics 18, 1210.

https://doi.org/10.1074/mcp.RA118.001104

Google Scholar: Author Only Title Only Author and Title

Chen, L. (2014) SWEET sugar transporters for phloem transport and pathogen nutrition. New Phytologist 201, $1150-1155$.

Google Scholar: Author Only Title Only Author and Title

Chen, L.-Q., Hou, B.-H., Lalonde, S., Takanaga, H., Hartung, M.L., Qu, X.-Q., Guo, W.-J., Kim, J.-G., Underwood, W., Chaudhuri, B. (2010) Sugar transporters for intercellular exchange and nutrition of pathogens. Nature 468, 527-532.

Google Scholar: Author Only Title Only Author and Title

Chen, L.-Q., Qu, X.-Q., Hou, B.-H., Sosso, D., Osorio, S., Fernie, AR., Frommer, W.B. (2012) Sucrose efflux mediated by SWEET proteins as a key step for phloem transport. Science 335, 207-211.

Google Scholar: Author Only Title Only Author and Title

Chen, X.-Y., Kim, J.-Y. (2009) Callose synthesis in higher plants. Plant Signaling \& Behavior 4, 489-492.

https://doi.org/10.4161/psb.4.6.8359

Google Scholar: Author Only Title Only Author and Title

De Marco, F., Pagliari, L., Degola, F., Buxa, S.V., Loschi, A, Dinant, S., Hir, R.L., Morin, H., Santi, S., Musetti, R. (2016) Combined microscopy and molecular analyses show phloem occlusions and cell wall modifications in tomato leaves in response to ' Candidatus Phytoplasma solani': response of tomato phloem to phytoplasma infection. Journal of Microscopy 263, 212-225.

https://doi.org/10.1111/jmi.12426

Google Scholar: Author Only Title Only Author and Title

De Marco, F., Batailler, B., Thorpe, M.R., Razan, F., Hir, R.L., Vilaine, F., Bouchereau, A, Martin-Magniette, M.-L., Eveillard, S., Dinant, S. (2020) Role of the SUT1 and SUT2 sugar transporters during stolbur phytoplasma infection in tomato (preprint). Plant Biology. https://doi.org/10.1101/2020.09.25.309708

Google Scholar: Author Only Title Only Author and Title

De Schepper, V., De Swaef, T., Bauweraerts, I., Steppe, K. (2013) Phloem transport: a review of mechanisms and controls. Journal of Experimental Botany 64, 4839-4850. https://doi.org/10.1093/jxb/ert302

Google Scholar: Author Only Title Only Author and Title

Dermastia, M. (2019) Plant hormones in phytoplasma infected plants. Front. Plant Sci. 10, 477. https://doi.org/10.3389/fpls.2019.00477

Google Scholar: Author Only Title Only Author and Title

Dodds, P.N., Lagudah, E.S. (2016) Starving the enemy. Science 354, 1377-1378.

Google Scholar: Author Only Title Only Author and Title

Doyle, JJ, Doyle, JL, 1990) DNA extraction from Arabidopsis. Focus 12, 13-15.

Durand, M., Mainson, D., Porcheron, B., Maurousset, L., Lemoine, R., Pourtau, N. (2018) Carbon source-sink relationship in

Arabidopsis thaliana: the role of sucrose transporters. Planta 247, 587-611. https://doi.org/10.1007/s00425-017-2807-4

Google Scholar: Author Only Title Only Author and Title

Ellinger, D., Voigt, C.A (2014) Callose biosynthesis in arabidopsis with a focus on pathogen response: what we have learned within the last decade. Annals of Botany 114, 1349-1358. https://doi.org/10.1093/aob/mcu120

Google Scholar: Author Only Title Only Author and Title

Ermacora, P., Osler, R. (2019) Symptoms of phytoplasma diseases, in: Phytoplasmas. Springer, pp. $53-67$.

Google Scholar: Author Only Title Only Author and Title

Eschrich, W. (1956) Kallose. Protoplasma 47, 487-530.

Google Scholar: Author Only Title Only Author and Title

Fatima, U., Senthil-Kumar, M. (2015) Plant and pathogen nutrient acquisition strategies. Frontiers in plant science 6, 750.

Google Scholar: Author Only Title Only Author and Title

Fotopoulos, V., Gilbert, M.J., Pittman, J.K., Marvier, AC., Buchanan, AJ., Sauer, N., Hall, J.L., Williams, L.E. (2003) The Monosaccharide Transporter Gene, AtSTP4, and the Cell-Wall Invertase, At $\beta$ fruct1, Are Induced in Arabidopsis during Infection with the Fungal 

available under aCC-BY-NC-ND 4.0 International license.

Biotroph Erysiphe cichoracearum. Plant Physiol. 132, 821-829. https://doi.org/10.1104/pp.103.021428

Google Scholar: Author Only Title Only Author and Title

Furch, AC., Hafke, J.B., Schulz, A, van Bel, AJ. (2007) Ca2+-mediated remote control of reversible sieve tube occlusion in Vicia faba. Journal of Experimental Botany 58, 2827-2838.

Google Scholar: Author Only Title Only Author and Title

Gould, N., Thorpe, M., Pritchard, J., Christeller, J., Williams, L., Roeb, G., Schurr, U., Minchin, P. (2012) AtSUC2 has a role for sucrose retrieval along the phloem pathway: evidence from carbon-11 tracer studies. Plant Science 188, 97-101.

Google Scholar: Author Only Title Only Author and Title

Hafke, J. B., van Amerongen, J. K., Kelling, F., Furch, A C., Gaupels, F., \& van Bel, A J. (2005) Thermodynamic battle for photosynthate acquisition between sieve tubes and adjoining parenchyma in transport phloem. Plant Physiology, 138(3), $1527-1537$.

Google Scholar: Author Only Title Only Author and Title

Hirose, T., Scofield, G.N., Terao, T. (2008) An expression analysis profile for the entire sucrose synthase gene family in rice. Plant Science 174, 534-543.

Google Scholar: Author Only Title Only Author and Title

Hong, Z, Zhang, Z, Olson, J.M., Verma, D.P.S. (2001) A novel UDP-glucose transferase is part of the callose synthase complex and interacts with phragmoplastin at the forming cell plate. The Plant Cell 13, 769-779.

Google Scholar: Author Only Title Only Author and Title

Horwitz, L. (1958) Some Simplified Mathematical Treatments of Translocation in Plants. Plant Physiology, $33(2), 81$.

Google Scholar: Author Only Title Only Author and Title

Huai, B., Yang, Q., Wei, X., Pan, Q., Kang, Z, Liu, J. (2020) TaSTP13 contributes to wheat susceptibility to stripe rust possibly by increasing cytoplasmic hexose concentration. BMC plant biology 20, 49.

Google Scholar: Author Only Title Only Author and Title

Julius, B.T., Leach, K.A, Tran, T.M., Mertz, R.A, Braun, D.M. (2017) Sugar Transporters in Plants: New Insights and Discoveries. Plant and Cell Physiology 58, 1442-1460. https://doi.org/10.1093/pcp/pcx090

Google Scholar: Author Only Title Only Author and Title

Le Hir, R., Spinner, L., Klemens, P.A, Chakraborti, D., de Marco, F., Vilaine, F., Wolff, N., Lemoine, R., Porcheron, B., Géry, C. (2015)

Disruption of the sugar transporters AtSWEET11 and AtSWEET12 affects vascular development and freezing tolerance in Arabidopsis.

Molecular Plant 8, 1687-1690.

Google Scholar: Author Only Title Only Author and Title

Lecourieux, F., Kappel, C., Lecourieux, D., Serrano, A, Torres, E., Arce-Johnson, P., Delrot, S. (2014) An update on sugar transport and signalling in grapevine. Journal of Experimental Botany 65, 821-832.

Google Scholar: Author Only Title Only Author and Title

Lee, D.-K., Ahn, S., Cho, H.Y., Yun, H.Y., Park, J.H., Lim, J., Lee, J., Kwon, S.W. (2016) Metabolic response induced by parasitic plantfungus interactions hinder amino sugar and nucleotide sugar metabolism in the host. Scientific reports 6, 1-11.

Google Scholar: Author Only Title Only Author and Title

Lee, I.-M., Martini, M., Bottner, K.D., Dane, R.A, Black, M.C., Troxclair, N. (2003) Ecological Implications froma Molecular Analysis of Phytoplasmas Involved in an Aster Yellows Epidemic in Various Crops in Texas. Phytopathology 93, $1368-1377$.

https://doi.org/10.1094/PHYTO.2003.93.11.1368

Google Scholar: Author Only Title Only Author and Title

Lemonnier, P., Gaillard, C., Veillet, F., Verbeke, J., Lemoine, R., Coutos-Thévenot, P., La Camera, S. (2014) Expression of Arabidopsis sugar transport protein STP13 differentially affects glucose transport activity and basal resistance to Botrytis cinerea. Plant molecular biology $85,473-484$.

Google Scholar: Author Only Title Only Author and Title

Levy, A, Guenoune-Gelbart, D., Epel, B.L. (2007) B-1,3-Glucanases: Plasmodesmal Gate Keepers for Intercellular Communication.

Plant Signaling \& Behavior 2, 404-407. https://doi.org/10.4161/psb.2.5.4334

Google Scholar: Author Only Title Only Author and Title

Li, H., Li, X., Xuan, Y., Jiang, J., Wei, Y., Piao, Z (2018) Genome wide identification and expression profiling of SWEET genes family reveals its role during Plasmodiophora brassicae-induced formation of clubroot in Brassica rapa. Frontiers in plant science $9,207$.

Google Scholar: Author Only Title Only Author and Title

Loewus, F.A, Kelly, S., Neufeld, E.F. (1962) Metabolism of myo-inositol in plants: conversion to pectin, hemicellulose, D-xylose, and sugar acids. Proceedings of the National Academy of Sciences of the United States of America 48, 421.

Google Scholar: Author Only Title Only Author and Title

Martini, M., Lee, I.-M., Bottner, K.D., Zhao, Y., Botti, S., Bertaccini, A, Harrison, N.A, Carraro, L., Marcone, C., Khan, AJ., Osler, R. (2007) Ribosomal protein gene-based phylogeny for finer differentiation and classification of phytoplasmas. International Journal of Systematic and Evolutionary Microbiology 57, 2037-2051. https://doi.org/10.1099/ijs.0.65013-0

Google Scholar: Author Only Title Only Author and Title 

available under aCC-BY-NC-ND 4.0 International license.

Martini, M., Musetti, R., Grisan, S., Polizzotto, R., Borselli, S., Pavan, F., Osler, R. (2009) DNA-Dependent Detection of the Grapevine Fungal Endophytes Aureobasidium pullulans and Epicoccum nigrum. Plant Disease 93, 993-998. https://doi.org/10.1094/PDIS-93-10-0993 Google Scholar: Author Only Title Only Author and Title

Maust, B.E., Espadas, F., Talavera, C., Aguilar, M., Santamaría, J.M., Oropeza, C. (2003) Changes in carbohydrate metabolism in coconut palms infected with the lethal yellowing phytoplasma. Phytopathology 93, 976-981.

Google Scholar: Author Only Title Only Author and Title

Meyer, S., Lauterbach, C., Niedermeier, M., Barth, I., Sjolund, R.D., Sauer, N. (2004) Wounding enhances expression of AtSUC3, a sucrose transporter from Arabidopsis sieve elements and sink tissues. Plant Physiology 134, 684-693.

Google Scholar: Author Only Title Only Author and Title

Milne, R.J., Grof, C.P., Patrick, J.W. (2018) Mechanisms of phloem unloading: shaped by cellular pathways, their conductances and sink function. Current opinion in plant biology 43, 8-15.

Google Scholar: Author Only Title Only Author and Title

Muller, P.Y., Janovjak, H., Miserez, AR., Dobbie, Z (2002) Short technical report processing of gene expression data generated by quantitative real-time RT-PCR. Biotechniques 32, 1372-1379.

Google Scholar: Author Only Title Only Author and Title

Musetti, R. (2010) Biochemical changes in plants infected by phytoplasmas. Phytoplasmas: Genomes, plant hosts and vectors $132-146$. Google Scholar: Author Only Title Only Author and Title

Musetti, R., Buxa, S.V., De Marco, F., Loschi, A, Polizzotto, R., Kogel, K.-H., van Bel, AJ.E. (2013) Phytoplasma-Triggered Ca 2+ Influx Is Involved in Sieve-Tube Blockage. MPMI 26, 379-386. https://doi.org/10.1094/MPMI-08-12-0207-R

Google Scholar: Author Only Title Only Author and Title

Musetti, R., Pagliari, L., Buxa, S.V., Degola, F., De Marco, F., Loschi, A, Kogel, K.-H., van Bel, AJ. (2016) OHMS**: phytoplasmas dictate changes in sieve-element ultrastructure to accommodate their requirements for nutrition, multiplication and translocation. Plant Signaling \& Behavior 11, e1138191.

Google Scholar: Author Only Title Only Author and Title

Namba, S. (2019) Molecular and biological properties of phytoplasmas. Proceedings of the Japan Academy, Series B 95, $401-418$.

Google Scholar: Author Only Title Only Author and Title

Nørholm, M.H., Nour-Eldin, H.H., Brodersen, P., Mundy, J., Halkier, B.A (2006) Expression of the Arabidopsis high-affinity hexose transporter STP13 correlates with programmed cell death. FEBS letters 580, 2381-2387.

Google Scholar: Author Only Title Only Author and Title

Oshima, K., Maejima, K., Namba, S. (2013) Genomic and evolutionary aspects of phytoplasmas. Front. Microbiol. 4. https://doi.org/10.3389/fmicb.2013.00230

Google Scholar: Author Only Title Only Author and Title

Pacifico, D., Galetto, L., Rashidi, M., Abbà, S., Palmano, S., Firrao, G., Bosco, D., Marzachì, C. (2015) Decreasing Global Transcript Levels over Time Suggest that Phytoplasma Cells Enter Stationary Phase during Plant and Insect Colonization. Appl. Environ.

Microbiol. 81, 2591-2602. https://doi.org/10.1128/AEM.03096-14

Google Scholar: Author Only Title Only Author and Title

Pagliari, L., Buoso, S., Santi, S., Furch, AC.U., Martini, M., Degola, F., Loschi, A, van Bel, AJ.E., Musetti, R. (2017) Filamentous sieve element proteins are able to limit phloem mass flow, but not phytoplasma spread. Journal of Experimental Botany $68,3673-3688$. https://doi.org/10.1093/jxb/erx199

Google Scholar: Author Only Title Only Author and Title

Pagliari, L., Martini, M., Loschi, A, Musetti, R. (2016) Looking inside phytoplasma-infected sieve elements: Acombined microscopy approach using Arabidopsis thaliana as a model plant. Micron 89, 87-97. https://doi.org/10.1016/j.micron.2016.07.007

Google Scholar: Author Only Title Only Author and Title

Pfaffl, M.W. (2001) A new mathematical model for relative quantification in real-time RT-PCR. Nucleic acids research 29, e45-e45.

Google Scholar: Author Only Title Only Author and Title

Pinheiro, J., Bates, D., DebRoy, S., Sarkar, D. (2018) R Core Team 2018 : linear and nonlinear mixed effects models. R package version 3.1-137. R Found. Stat. Comput. Retrieved from https://CRAN. R-project. org/package= nlme (accessed 19 Jul. 2018).

Google Scholar: Author Only Title Only Author and Title

Prezelj, N., Covington, E., Roitsch, T., Gruden, K., Fragner, L., Weckwerth, W., Chersicola, M., Vodopivec, M., Dermastia, M. (2016) Metabolic consequences of infection of grapevine (Vitis vinifera L.) cv."Modra frankinja" with Flavescence Dorée phytoplasma.

Frontiers in Plant Science 7, 711.

Google Scholar: Author Only Title Only Author and Title

Roitsch, T., González, M.-C. (2004) Function and regulation of plant invertases: sweet sensations. Trends in plant science 9, $606-613$. Google Scholar: Author Only Title Only Author and Title

Rojas, C.M., Senthil-Kumar, M., Tzin, V., Mysore, K. (2014) Regulation of primary plant metabolism during plant-pathogen interactions 

available under aCC-BY-NC-ND 4.0 International license.

and its contribution to plant defense. Frontiers in plant science 5, 17.

Google Scholar: Author Only Title Only Author and Title

Ruan, Y.-L. (2014) Sucrose Metabolism: Gateway to Diverse Carbon Use and Sugar Signaling. Annu. Rev. Plant Biol. 65, 33-67. https://doi.org/10.1146/annurev-arplant-050213-040251

Google Scholar: Author Only Title Only Author and Title

Sager, R.E., Lee, J.-Y. (2018) Plasmodesmata at a glance. Journal of Cell Science 131.

Google Scholar: Author Only Title Only Author and Title

Santi, S., De Marco, F., Polizzotto, R., Grisan, S., Musetti, R. (2013a. Recovery from stolbur disease in grapevine involves changes in sugar transport and metabolism. Front. Plant Sci. 4. https://doi.org/10.3389/fpls.2013.00171

Google Scholar: Author Only Title Only Author and Title

Santi, S., Grisan, S., Pierasco, A, De Marco, F., Musetti, R. (2013b. Laser microdissection of grapevine leaf phloem infected by stolbur reveals site-specific gene responses associated to sucrose transport and metabolism: LM of stolbur-infected grapevine phloem

Plant, Cell \& Environment 36, 343-355. https://doi.org/10.1111/j.1365-3040.2012.02577.x

Google Scholar: Author Only Title Only Author and Title

Schindelin, J., Arganda-Carreras, I., Frise, E., Kaynig, V., Longair, M., Pietzsch, T., Preibisch, S., Rueden, C., Saalfeld, S., Schmid, B., Tinevez, J.-Y., White, D.J., Hartenstein, V., Eliceiri, K., Tomancak, P., Cardona, A (2012) Fiji: an open-source platform for biologicalimage analysis. Nature Methods 9, 676-682. https://doi.org/10.1038/nmeth.2019

Google Scholar: Author Only Title Only Author and Title

Schneider, R., Hanak, T., Persson, S., Voigt, C.A (2016) Cellulose and callose synthesis and organization in focus, what's new? Current Opinion in Plant Biology 34, 9-16. https://doi.org/10.1016/j.pbi.2016.07.007

Google Scholar: Author Only Title Only Author and Title

Sherson, S.M., Alford, H.L., Forbes, S.M., Wallace, G., Smith, S.M. (2003) Roles of cell-wall invertases and monosaccharide transporters in the growth and development of Arabidopsis. Journal of Experimental Botany 54, 525-531. https://doi.org/10.1093/jxb/erg055

Google Scholar: Author Only Title Only Author and Title

Stein, O., Granot, D. (2019) An Overview of Sucrose Synthases in Plants. Front. Plant Sci. 10, 95. https://doi.org/10.3389/fpls.2019.00095 Google Scholar: Author Only Title Only Author and Title

Tadege, M., Bucher, M., Stähli, W., Suter, M., Dupuis, I., Kuhlemeier, C. (1998) Activation of plant defense responses and sugar efflux by expression of pyruvate decarboxylase in potato leaves. The Plant Journal 16, 661-671.

Google Scholar: Author Only Title Only Author and Title

Tan, T.-C., Kracher, D., Gandini, R., Sygmund, C., Kittl, R., Haltrich, D., Hällberg, B.M., Ludwig, R., Divne, C. (2015) Structural basis for cellobiose dehydrogenase action during oxidative cellulose degradation. Nature communications 6, 7542.

Google Scholar: Author Only Title Only Author and Title

van Bel, AJ.E., Musetti, R. (2019) Sieve element biology provides leads for research on phytoplasma lifestyle in plant hosts. Journal of Experimental Botany 70, 3737-3755. https://doi.org/10.1093/jxb/erz172

Google Scholar: Author Only Title Only Author and Title

Verma, D.P.S., Hong, Z (2001) Plant callose synthase complexes 9.

Vincent, C., Minchin, P.E.H., Liesche, J. (2019) Noninvasive Determination of Phloem Transport Speed with Carbon-14 (14C), in: Liesche, J. (Ed.), Phloem, Methods in Molecular Biology. Springer New York, New York, NY, pp. 153-162. https://doi.org/10.1007/978-14939-9562-2_13

Google Scholar: Author Only Title Only Author and Title

Will, T., van Bel, AJ. (2006) Physical and chemical interactions between aphids and plants. Journal of experimental botany $57,729-737$. Google Scholar: Author Only Title Only Author and Title

Wipf, D., Pfister, C., Mounier, A, Leborgne-Castel, N., Frommer, W.B., Courty, P.-E. (2020) Identification of Putative Interactors of Arabidopsis Sugar Transporters. Trends in Plant Science.

Google Scholar: Author Only Title Only Author and Title

Wu, S.-W., Kumar, R., Iswanto, AB.B., Kim, J.-Y. (2018) Callose balancing at plasmodesmata. Journal of Experimental Botany. https://doi.org/10.1093/jxb/ery317

Google Scholar: Author Only Title Only Author and Title

Xie, B., Hong, Z (2011) Unplugging the callose plug from sieve pores. Plant Signaling \& Behavior 6, $491-493$. https://doi.org/10.4161/psb.6.4.14653

Google Scholar: Author Only Title Only Author and Title

Xie, B., Wang, X., Zhu, M., Zhang, Z, Hong, Z (2011) CalS7 encodes a callose synthase responsible for callose deposition in the phloem: A phloem-specific callose synthase. The Plant Journal 65, 1-14. https://doi.org/10.1111/j.1365-313X.2010.04399.x

Google Scholar: Author Only Title Only Author and Title

Yamada, K., Kanai, M., Osakabe, Y., Ohiraki, H., Shinozaki, K., Yamaguchi-Shinozaki, K. (2011) Monosaccharide Absorption Activity of 
bioRxiv preprint doi: https://doi.org/10.1101/2021.06 25.449948; this version posted June 26, 2021. The copyright holder for this preprint (which was not certified by peer review) is the author/funder, who has granted bioRxiv a license to display the preprint in perpetuity. It is made available under aCC-BY-NC-ND 4.0 International license.

Arabidopsis Roots Depends on Expression Profiles of Transporter Genes under High Salinity Conditions. J. Biol. Chem 286, 4357743586. https://doi.org/10.1074/jbc.M111.269712

Google Scholar: Author Only Title Only Author and Title

Yao, D., Gonzales-Vigil, E., Mansfield, S.D. (2020) Arabidopsis sucrose synthase localization indicates a primary role in sucrose translocation in phloem. Journal of experimental botany 71, 1858-1869.

Google Scholar: Author Only Title Only Author and Title

Yao, L., Yu, Q., Huang, M., Hung, W., Grosser, J., Chen, S., Wang, Y., Gmitter, F.G. (2019) Proteomic and metabolomic analyses provide insight into the off-flavour of fruits from citrus trees infected with 'Candidatus Liberibacter asiaticus.' Horticulture research 6, 1-13.

Google Scholar: Author Only Title Only Author and Title

Zavaliev, R., Epel, B.L. (2015) Imaging Callose at Plasmodesmata Using Aniline Blue: Quantitative Confocal Microscopy, in: Heinlein, M. (Ed.), Plasmodesmata, Methods in Molecular Biology. Springer New York, New York, NY, pp. 105-119. https://doi.org/10.1007/978-14939-1523-1_7

Google Scholar: Author Only Title Only Author and Title

Zhang, C. (2018) Mechanisms of phloem loading. Current Opinion in Plant Biology 5.

Google Scholar: Author Only Title Only Author and Title 